\title{
MILP Formulation Improvement with $k$-Means Clustering for the Beam Layout Optimization in Multibeam Satellite Systems
}

\author{
Jean-Thomas Camino ${ }^{\mathrm{a}, \mathrm{b}}$, Christian Artigues ${ }^{\mathrm{a}}$, Laurent Houssin ${ }^{\mathrm{a}, *}$, Stéphane Mourgues ${ }^{\mathrm{b}}$ \\ ${ }^{a}$ CNRS ; LAAS ; 7 avenue du colonel Roche, F-31077 Toulouse, France \\ ${ }^{b}$ Airbus Defence and Space, Space Systems, Telecommunication Systems Department, 31 Rue des Cosmonautes, F-31402 Toulouse, France
}

\begin{abstract}
This paper addresses an NP-hard design optimization problem in a multibeam satellite communication system. This problem consists in designing irregular beam layouts to satisfy non-uniform user traffic demands over the satellite service area, under antenna constraints, satellite payload constraints and a telecommunication mission criterion. Efficiently solving this problem is of crucial importance due to its impact on the system performance and cost. We compare three mixed-integer linear programming formulations. The first one, issued from previous work, is based on a linearization of both convex and non-convex Euclidean distance constraints. The two other aim at reducing the solution space size and at breaking symmetry inherent to the first formulation. For that purpose, we introduce a new process to interface $k$-means clustering with mixed-integer linear programming. We examine an exact and a heuristic approach for exploiting these principles that yield two new formulations. The heuristic approach outperforms the others based on our tests on a set of large-scale realistic problem instances, allowing to use mixed-integer linear programming in the industrial context.
\end{abstract}

Keywords: Mixed Integer Linear Programming, Multibeam Satellite Systems Design, K-means clustering

\section{Introduction}

In this paper, we consider a design optimization problem for a multibeam satellite communication system illustrated in Fig. 1. Roughly, one or several gateway stations send radio frequency signals to the satellite through the uplink (1). There is a service area on which end-user terminals, the recipients of the carried information (called the "stations" in this paper), are located. The satellite payload (2) receives, converts, and amplifies the signals received from the gateways and retransmit them in the different beams of the downlink (3) in such a way that an end-user station must be covered by a beam to receive the required information from the satellite. A beam refers to directional antennas, also called beam or high gain antennas. Basically, a high gain antenna can concentrate the radiation in a specific direction, which allows to communicate with users located in a narrow circular zone on earth defined by a beam. In telecommunication satellites, several beams can be formed simultaneously and positioned on demand to different areas on earth. The presence of multiple narrow beams allow to better concentrate the antenna gain and to better reuse the expensive bandwidth resource. Because of the increasing demand in various data exchanges, there has been a significant amount of research devoted to performance improvement of such systems, as reviewed in [22], such as the use of optical link techniques [19], the coordination of two co-localized satellites [7] or the design of multibeam array antennas [21]. The literature on solution approaches for power and frequency allocation problems is rather rich $[18,11,12,23,24,1,14,10]$. However, papers dealing with beam layout algorithms are not frequently published. In this paper we consider the NP-hard integrated beam layout and reflector allocation optimization problem previously considered in $[4,5]$. Each user station in the service area is defined by its $(x, y)$ coordinates in a projected 2D space and a traffic demand. A user station can be covered by a beam, or left uncovered. A beam is defined by its center and a

\footnotetext{
${ }^{*}$ Corresponding author

Email addresses: jean-thomas.camino@airbus.com (Jean-Thomas Camino), artigues@laas.fr(Christian Artigues), houssin@laas.fr (Laurent Houssin), stephane.mourgues@airbus.com (Stéphane Mourgues)
} 
diameter. The center can be positioned continuously on the surface, while the diameter has to be selected in a discrete set. A beam must be assigned an antenna reflector, among a limited available number such reflectors, which are the device that redirects the electromagnetic energy to form the beam on earth. The number of used beams cannot exceed a maximum number. Each beam can cover one or several stations located inside its diameter up to a limited traffic capacity that depends on the selected diameter: the larger the diameter, the lower the capacity. There are minimum distance separation constraints between any two beams and the constraints are tighter for two beams assigned to the same reflector. The objective is to maximize the covered traffic under the above-defined constraints. In Fig. 1, 13 beams are used to cover the service area. The colors of the beams represent the assigned reflectors. The limited beam overlapping illustrates the separation constraint that exists between any two beams. Observe that the distance between two beam centers is larger if the two beams are assigned to the same reflector. In this case the separation constraint is stronger.

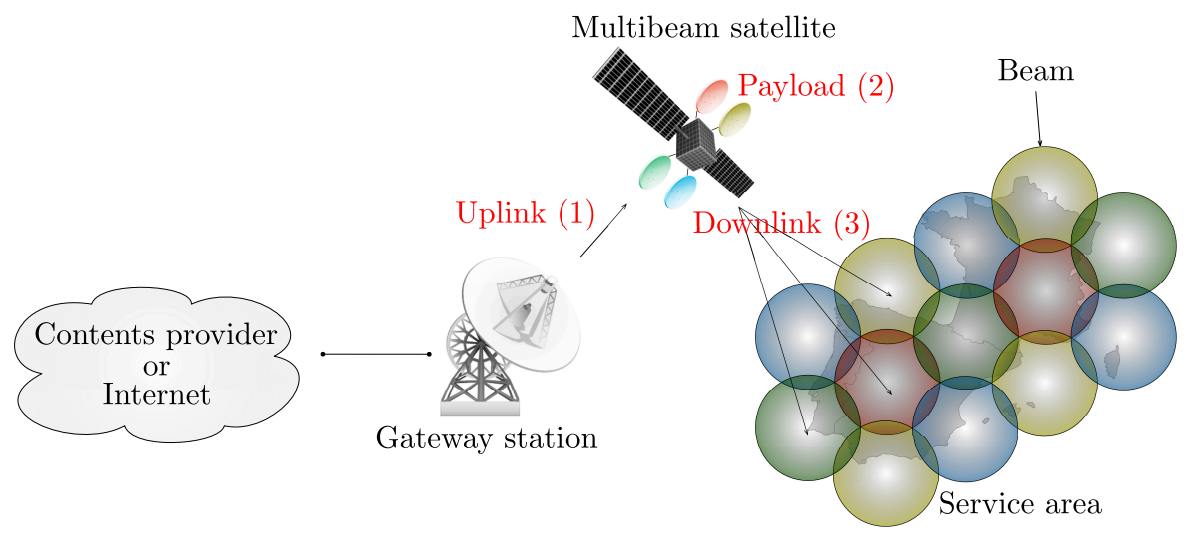

Figure 1: Forward link of a multibeam satellite system

Another example displayed in Fig. 2 and taken from [4] illustrates the interest of continuous positioning of the beams and diameter selection. On the left side with subfigures (a), (c) and (e), three different beam layouts and antenna reflector assignments represented by the different colors are used to cover the same service area. Beam layout (a) is a regular beam layout as used in practice where 117 beams are needed to cover the area. Beam layout (c) is also a regular beam layout of 50 beams where each beam has the same diameter, greater than (a). Beam layout (e) is a non-regular beam layout with different diameters such that only 48 beams are needed to cover the area. On the right side with sub-figures (b), (d) and (f), the traffic covered by each beam layout is represented via another color encoding. Blue and light blue beams cover low and medium traffic demands while yellow, orange and red beams cover high, very high and excessive traffic demands, respectively. The figure shows that the regular beam layout $(a, b)$ is inefficient: the large number of blue beams means that many beams are underused and, on the other hand, there is an excessive covered demand for the red beam. The beam layouts $(c, d)$ and $(e, f)$ require much less underused beams, which gives a more cost-effective solution in practice. Furthermore, using different diameters (e,f) avoids overused beams since it allows to better adjust the capacity to the demand. In fact, the beam layout $(\mathrm{a}, \mathrm{b})$ follows the so-called hexagonal lattice pattern with a single beam width. This is a close-to-optimal beam layout when the traffic demands are homogeneous over the service area as it maximizes the circle packing density [4, 6]. However, the Fig. 2 example shows that this is no more the case for heterogeneous traffic demands. Using different beam diameters and irregular beam layout yields better solutions.

In terms of computational complexity, this problem was shown to be NP-hard in [5]. It mixes discrete variables (assignment of reflectors, diameters ${ }^{1}$ and stations to beams) and continuous variables (coordinates of the beams) linked by convex constraints (maximum distance between the beam center and a covered station) and non convex constraints (minimum distance between two beams).

\footnotetext{
${ }^{1}$ For reasons explained in [4], it is not technically possible to adjust the beam diameter continuously.
} 


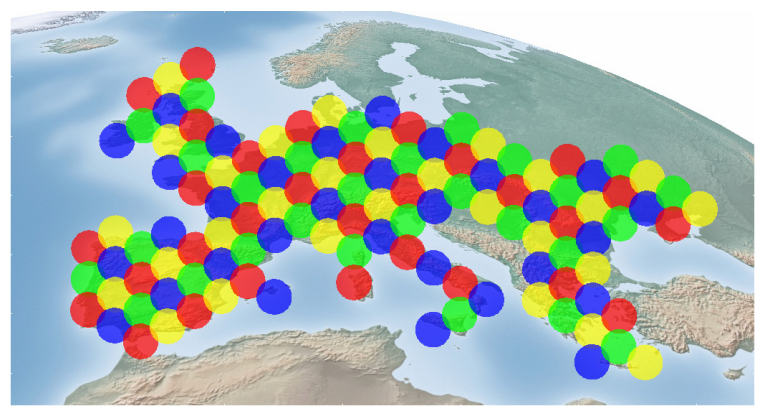

(a)

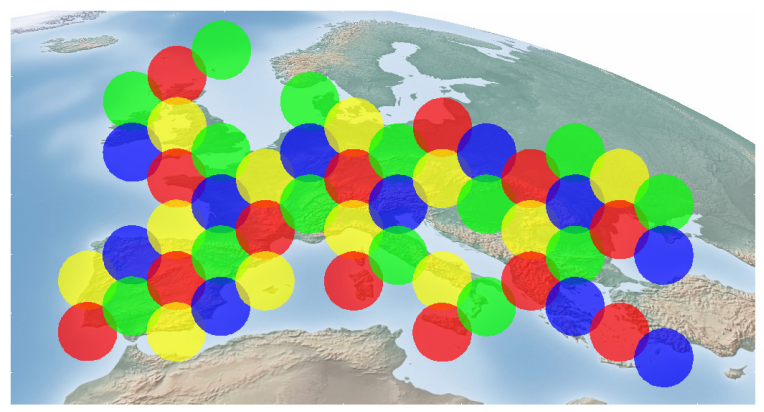

(c)

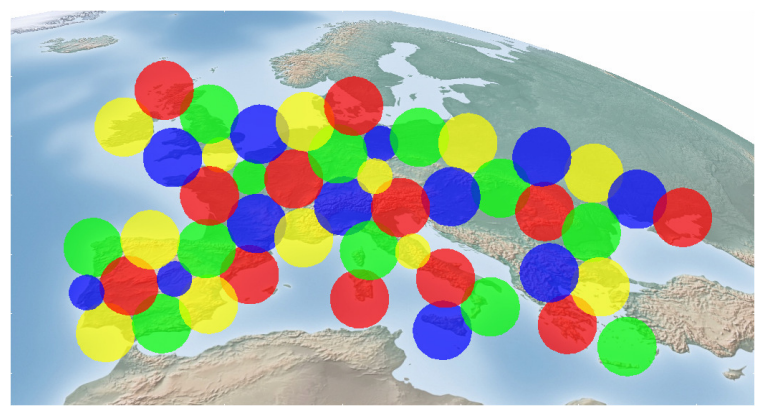

(e)

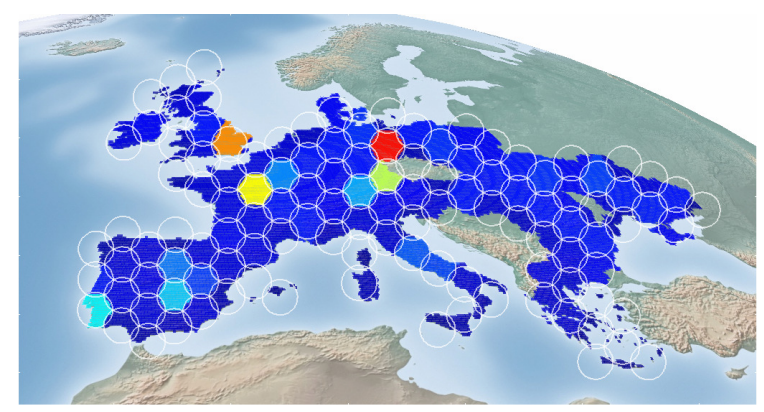

(b)

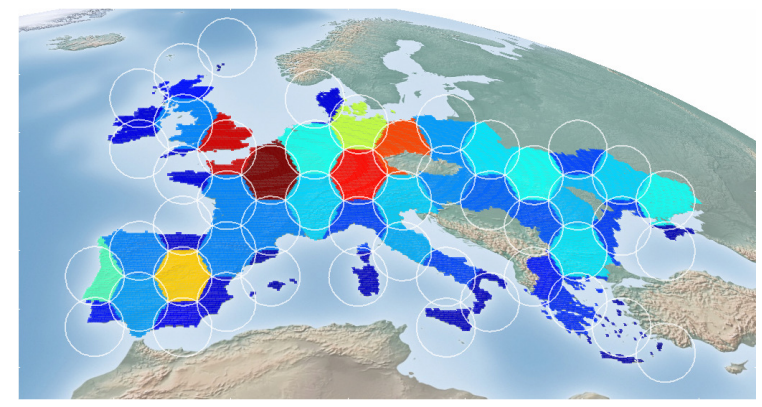

(d)

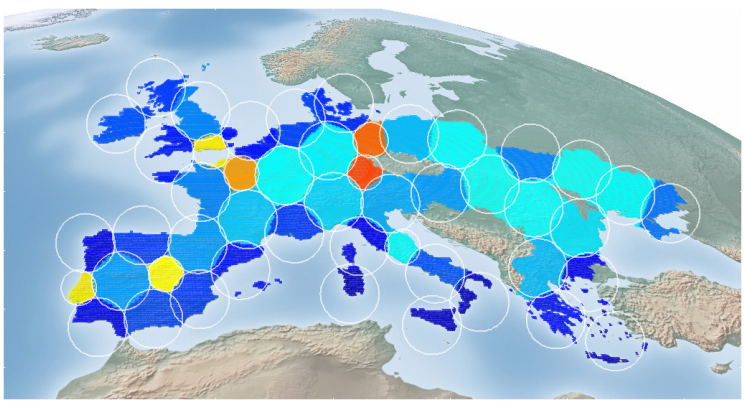

(f)

Figure 2: (a),(c),(e) Satellite reflector mapping: 117 regular beams, 50 regular beams, 48 irregular beams (b),(d),(f) Load per beam: 117 regular beams, 50 regular beams, 48 irregular beams (blue $=$ low values, red $=$ high values)

Different approaches previously aimed at obtaining irregular beam layouts in the literature. In [2], an approach based on partitioning the area into polygons of balanced load is proposed without taking the reflector allocation problem into account. In [8], the authors propose to tackle the beam layout subproblem and the reflector allocation problem sequentially, which frequently yields infeasibility. Indeed, as remarked in [3,4], it is likely that a layout optimized independently of the reflector allocation yields infeasibility due to the reflector allocation-dependent separation constraints. To overcome this issue, a randomized greedy procedure coupled with a graph coloring heuristic was proposed in [3] to solve the two subproblems jointly. The main issue with this approach is that the set of possible positions for the beam centers needs to be discretized, which yields suboptimal solutions. In [5], a mixed-integer linear program (MILP) was proposed to solve the integrated beam layout and reflector assignment problem with continuous variables representing the coordinate of the beam centers. To obtain the MILP, the approach uses a linearization technique of both convex and non convex euclidean distance constraints. In [4], the merits of this mixed discrete/continuous approach compared to the pure discrete approach of [5] was discussed. However, the proposed MILP was unable to obtain competitive results on large problem instances due to the explosion of binary variables for large-instances and the intrinsic symmetries. 
The contributions of this paper are to propose several approaches to reduce the MILP size, in terms of binary variables and constraints, to improve its performance on large problem instances. A first contribution is to propose a way to compute an upper bound of the number of necessary beams. Indeed, Having a reduced maximum number of beams indeed reduces the MILP size. A second contribution is to partition the user stations in clusters and to assign each beam to a single cluster, so as to reduce symmetries and possibly reduce the number of variable and constraints. We use the $k$-means clustering techniques to achieve this goal. We propose to limit the number of beams per cluster in two way. An exact approach preserves the generality of the formulation in the sense that the resulting MILP maps the same set of solutions as the original MILP. A heuristic approach performs more variable and constraint reductions but yields a MILP whose feasible region is only a subset of the feasible region of the original MILP, which possibly lose optimality. Significant reductions are obtained by the latter approach that obtains in addition much better results than the original MILP in terms of demand coverage on large instances.

The rest of the article is structured as follows. In Section 2, we provide a non-linear formulation of the problem and we recall the initial mixed-integer programming formulation of the problem presented in [5]. In Section 3, we propose a new upper bound on the number of necessary beams. Several clustering-based problem size reduction techniques for solving larger problem instances are then introduced in Section 4. All approaches are experimentally compared in Section 5 to illustrate the efficiency of the proposed approach on instances generated according to realistic scenarios established by the Airbus Defense and Space Telecommunication Systems Department. Concluding remarks are drawn in Section 6.

\section{Problem description and initial mixed-integer linear programming formulation}

\subsection{Non-linear formulation and problem complexity}

The goal of this section is to provide a generic description of the optimization problem and to introduce notation for the several problem parameters. Let us recall that the problem consists of defining the $(x, y)$ coordinates and the diameters (also called beamwidths) of a certain maximum number $N_{B}$ of beams, and associating them to user stations and to antenna reflectors. This set of beams will be denoted by $\mathcal{B}=\left\{1, \cdots, N_{B}\right\}$. Some of them can be set to be "non-active": the number of active beams determines the actual number of beams that would be embarked on the spacecraft. A binary variable $a_{b}$ is introduced for each beam $b \in \mathcal{B}$ such that beam $b$ is active if $a_{b}=1$. With these beams, the goal is to provide service to $N_{S}$ user stations positioned on the coverage area. Each user station $s$ in the set of stations $\mathcal{S}=\left\{1, \cdots, N_{S}\right\}$ is characterized by its own traffic demand $T_{S} \in \mathbb{R}^{+}$(in Megabits per second) and its coordinates $S_{\text {coord }, s}=\left(X_{\text {stations }, s}, Y_{\text {stations }, s}\right) \in \mathbb{R}^{2}$ in the service area. The problem is to maximize the aggregate covered traffic. We assume that $N_{W}$ possible beamwidths $W_{1}, \cdots, W_{N_{W}} \in \mathbb{R}^{+}$are defined in advance. Indeed, having continuous beamwidth variables is not an industrially viable option since each distinct beamwidth corresponds to a different radiofrequency feed design. Hence, offering a large number of different beamwidths would be too costly. In the rest of the paper, the index set $\left\{1, \cdots, N_{W}\right\}$ will be denoted by $\mathcal{W}$. We also assume that there are $N_{R}$ reflectors available on the satellite payload, defining the set $\mathcal{R}=\left\{1, \cdots, N_{R}\right\}$. We denote by $N_{\min }$ the minimum number of stations that must be covered by each beam placed on the coverage. Finally, for each beamwidth $\omega \in \mathcal{W}$, we define a load upper bound $\Gamma_{w}$. All these input parameters allow to define the following non-linear beam layout optimization problem formulation (1-11) issued from [5].

$$
\operatorname{Maximize} \sum_{(s, b) \in \mathcal{S} \times \mathcal{B}} T_{s} \alpha_{s, b}
$$

under the following constraints

$$
\begin{aligned}
& \forall s \in \mathcal{S}, \quad \sum_{b \in \mathcal{B}} \alpha_{s, b} \leq 1 \\
& \forall b \in \mathcal{B}, \quad \sum_{w \in \mathcal{W}} \omega_{b, w}=1
\end{aligned}
$$




$$
\begin{gathered}
\forall s \in \mathcal{S}, \forall b \in \mathcal{B}, \sqrt{\left(x_{b}-X_{\text {stations }, s}\right)^{2}+\left(y_{b}-Y_{\text {stations }, s}\right)^{2}} \leq \frac{1}{2} \sum_{w \in \mathcal{W}} W_{w} \omega_{b, w}+\left(1-\alpha_{s, b}\right) M_{s} \\
\forall b \in \mathcal{B}, \quad \sum_{r \in \mathcal{R}} \rho_{b, r}=1
\end{gathered}
$$$$
\forall b, b^{\prime} \in \mathcal{B} \text { such that } b^{\prime}>b,
$$$$
\sqrt{\left(x_{b^{\prime}}-x_{b}\right)^{2}+\left(y_{b^{\prime}}-y_{b}\right)^{2}} \geq \frac{1}{2}\left(\sum_{w \in \mathcal{W}} W_{w} \omega_{b, w}+\sum_{w \in \mathcal{W}} W_{w} \omega_{b^{\prime}, w}\right) \varepsilon+\lambda_{b, b^{\prime}}
$$$$
\forall b, b^{\prime} \in \mathcal{B} \text { such that } b^{\prime}>b, \forall r \in \mathcal{R}, \quad \beta_{b, b^{\prime}}+\rho_{b, r}+\rho_{b^{\prime}, r} \leq 2+\left(1-a_{b}\right)+\left(1-a_{b^{\prime}}\right)
$$$$
\forall b, b^{\prime} \in \mathcal{B} \text { such that } b^{\prime}>b, \quad \lambda_{b, b^{\prime}} \geq \frac{\kappa-\varepsilon}{2} \sum_{w \in \mathcal{W}} W_{w}\left(\omega_{b, w}+\omega_{b^{\prime}, w}\right)-(\kappa-\varepsilon) \max _{w \in \mathcal{W}} W_{w} \beta_{b, b^{\prime}}
$$$$
\forall b \in \mathcal{B}, \quad \sum_{s \in \mathcal{S}} \alpha_{s, b} \leq N_{s} a_{b}
$$$$
\forall b \in \mathcal{B}, \quad \sum_{s \in \mathcal{S}} T_{s} \alpha_{s, b} \leq \sum_{w \in \mathcal{W}} \Gamma_{w} \omega_{b, w}
$$$$
\forall b \in \mathcal{B}, \quad \sum_{s \in \mathcal{S}} \alpha_{s, b} \geq N_{\min } a_{b}
$$

Variables: $\alpha_{s, b}, \omega_{b, w}, \rho_{b, r}, a_{b}, \beta_{b, b^{\prime}} \in\{0,1\}, x_{b}, y_{b} \in \mathbb{R}$, and $\lambda_{b, b^{\prime}} \in \mathbb{R}^{+}$

To handle the optimization objective in practice, for all $s \in \mathcal{S}$ and $b \in \mathcal{B}$, the $\alpha_{s, b} \in\{0,1\}$ variable is defined and characterizes the potential allocation of the station $s$ to the beam $b: \alpha_{s, b}=1$ if and only if $b$ serves $s$. It allows to express linearly the objective of the problem where $T_{s}$ is the individual demand of station $s$, as shown in equation (1). Note that we assume that a station can be associated to at most one beam, leading to the set of constraints (2).

Each beam $b \in \mathcal{B}$ must be assigned exactly one beamwidth $w \in \mathcal{W}$, this allocation being dealt with by the variables $\omega_{b, w} \in\{0,1\}$ and the constraints (3).

For each beam $b \in \mathcal{B}, x_{b} \in \mathbb{R}$ and $y_{b} \in \mathbb{R}$ are variables corresponding to the coordinates of its beam center. For $s \in \mathcal{S}$ and $b \in \mathcal{B}$, if $\alpha_{s, b}=1$, then the beam $b$ must cover geometrically the station $s$, which is expressed by constraints (4) stating that the distance between the station and the beam center must be lower than the selected beam radius. If now $\alpha_{s, b}=0$, then $M_{s} \geq 0$ is needed to relax these geometrical covering constraints. To avoid detrimental numerical behaviors, it is desirable to choose a value for $M_{s}$ as low as possible, but on the other hand, it must be sufficiently high so as to preserve the possibility for the considered beam to choose beam centers that allow to cover any other station in every way possible: any station should be able to be coincident with any point of the disk representing that beam, whatever its diameter. The lowest value for $M_{s}$ that allows to verify this last point is $M_{s}=\max _{s^{\prime} \in \mathcal{S}}\left\|S_{\text {coord }, s^{\prime}}-S_{\text {coord }, s}\right\|$. Indeed, an active beam can not be positioned, relatively to a station $s$, farther than the farthest station from $s$ plus the beam radius. By definition, there is no station to cover at a larger distance. To indicate that each beam must be assigned to exactly one reflector, we introduce for all $b \in \mathcal{B}$ and for all $r \in \mathcal{R}$ the allocation variable $\rho_{b, r} \in\{0,1\}$ and the corresponding constraints (5).

Let us now define $\kappa$ and $\varepsilon$ as angular separation coefficients for two active beam centers but the coefficient $\kappa$ only applies when the two beams are associated with the same reflector whereas $\varepsilon$ always applies for any two active beams. Furthermore, we have $\kappa>\varepsilon$ as the separation constraint between two beam centers is stronger if the two beams are 


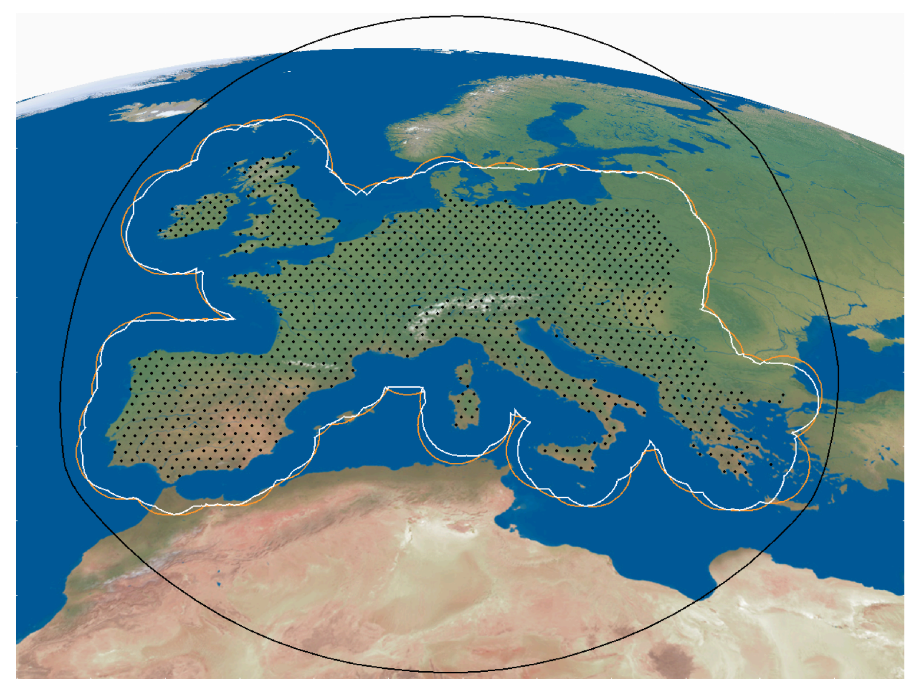

Figure 3: Continuous beam center domains implied by constraints (12) in black and by constraints $(11)$ in orange $\left(N_{\text {min }}=1\right)$ and in white $\left(N_{\text {min }}=2\right)$

associated with the same reflector. In practice, we have $\kappa \in\left[\frac{3}{2}, \sqrt{3}\right]$ and $\varepsilon \in\left[\frac{1}{4}, \frac{\sqrt{3}}{2}\right]$. For any two beams $b$ and $b^{\prime}$, their centers must be distant from each other by their mean radius times $\varepsilon$, which is ensured by constraints (6). Note that an additional variable $\lambda_{b, b^{\prime}}$ appear in the right hand side of the constraints. This variable is equal to $\kappa-\varepsilon$ times the mean radius if, in addition, the two beams share the same reflector and to 0 otherwise. For $b, b^{\prime} \in \mathcal{B}$ such that $b^{\prime}>b$, we define the binary variables $\beta_{b, b^{\prime}} \in\{0,1\}$ such that if $b$ and $b^{\prime}$ are active beams that use the same reflector then $\beta_{b, b^{\prime}}=0$. This is stated by constraints (7). Then, constraints (8) enforce $\lambda_{b, b^{\prime}}$ to be at least $\kappa-\varepsilon$ times the mean radius of $b$ and $b^{\prime}$ if $\beta_{b, b^{\prime}}=0$. If $\beta_{b, b^{\prime}}=1$, the constraint is discarded.

In the remainder of the paper, to distinguish the two families of separation constraints, the separation constraints for active beams associated with the same reflector will be called "antenna" constraints while the less tight separation constraints for any two active beams will be called the "non overlapping constraints". We refer to [4] for a technological justification of these constraints.

The interaction of non-active beams with user stations still needs to be suppressed at this point: a beam that is not active cannot cover any station. This is expressed with the constraints (9).

The constraints (10) express that a beam cannot be assigned more traffic than a certain limit $\Gamma_{w}$ defined per beamwidth.

The last constraint set (11) makes it necessary for any active beam to cover at least $N_{\min } \in \mathbb{N}$ stations, that minimum number being a tunable input parameter for the mathematical model. In practice, we will often work with at least $N_{\min }=1$. Note that these constraints directly impact the continuous domain of possible beam centers, just like the constraints (4), by removing this time all the centers from which no station is reachable whatever the beamwidth. Let $\mathcal{A}$ be the domain of beam centers allowed by constraints (4) and $\mathcal{A}^{\prime}$ the one resulting from constraints (11). On Fig. 3, $\mathcal{A}$ is the set delimited by the black line, while $\mathcal{A}^{\prime}$ is represented twice in orange and in white for $N_{\min }=1$ and $N_{\min }=2$ respectively. Note that we can verify on Fig. 3 that the goal we had above when defining the $M_{S}$ values to preserve the ability to cover any station in all the possible ways has indeed been reached: it is materialized by the fact that $\mathcal{A}^{\prime} \subset \mathcal{A}$.

In [5], the problem was proven NP-hard by reduction of the circle covering problem. 


\subsection{Initial mixed integer linear programming model}

In this section, the MILP formulation presented in [5] is recalled using the notation of section 2.1 for the problem parameters: the formulation is given by constraints (1-11), except that non linear constraints (4) and (6) are replaced by constraints (12-14) below. This formulation will be referred to as formulation MILP1. The formulation is based on a new linearization technique presented in [5] and that was recently reused for other optimization problems under Euclidean constraints [20, 26, 27].

$$
\begin{gathered}
\forall s \in \mathcal{S}, \forall b \in \mathcal{B}, \forall u \in \mathcal{U}, \quad\left(\begin{array}{l}
x_{b}-X_{\text {stations }, s} \\
y_{b}-Y_{\text {stations }, s}
\end{array}\right)^{T}\left(\begin{array}{l}
U_{u, x} \\
U_{u, y}
\end{array}\right) \leq \frac{1}{2} \sum_{w \in \mathcal{W}} W_{w} \omega_{b, w}+\left(1-\alpha_{s, b}\right) M_{S} \\
\forall b, b^{\prime} \in \mathcal{B} \text { such that } b^{\prime}>b, \quad \sum_{u \in \mathcal{U}} \gamma_{b, b^{\prime}, u} \geq a_{b}+a_{b^{\prime}}-1 \\
\forall b, b^{\prime} \in \mathcal{B} \text { such that } b^{\prime}>b, \forall u \in \mathcal{U}, \\
\left(\begin{array}{l}
x_{b^{\prime}}-x_{b} \\
y_{b^{\prime}}-y_{b}
\end{array}\right)^{T}\left(\begin{array}{l}
U_{u, x} \\
U_{u, y}
\end{array}\right) \geq \\
\frac{1}{2}\left(\sum_{w \in \mathcal{W}} W_{w} \omega_{b, w}+\sum_{w \in \mathcal{W}} W_{w} \omega_{b^{\prime}, w}\right) \varepsilon+\lambda_{b, b^{\prime}}-N\left(1-\gamma_{b, b^{\prime}, u}\right)
\end{gathered}
$$

Additional variables: $\gamma_{b, b^{\prime}, u} \in\{0,1\}$

Formulation MILP1 is an approximation of the non-linear problem (1-11), as detailed in the article [5]. The approximation comes from the linearization of Euclidean distance constraints using the principle of uniform discretization of the directions of the plane. New parameters, namely a number of directions $n_{\text {directions }}$ and a set of unit vectors (directions) $U_{u}$ for $u \in \mathcal{U}=\left\{1, \ldots, n_{\text {directions }}\right\}$ are needed for this linearization, where:

$$
U_{u}=\left(\begin{array}{l}
U_{u, x} \\
U_{u, y}
\end{array}\right)=\left(\begin{array}{c}
\cos \left(\frac{2(u-1) \pi}{n_{\text {directions }}}\right) \\
\sin \left(\frac{2(u-1) \pi}{n_{\text {directions }}}\right)
\end{array}\right) \in \mathbb{R}^{2}
$$

In Fig. 4(a), a set of 8 directions are considered. As illustrated in Fig. 4(b), these directions allow to define a polyhedral overapproximation $\mathcal{P}$ and a polyhedral underapproximation $\mathcal{P}^{\prime}$ of the unit circle.

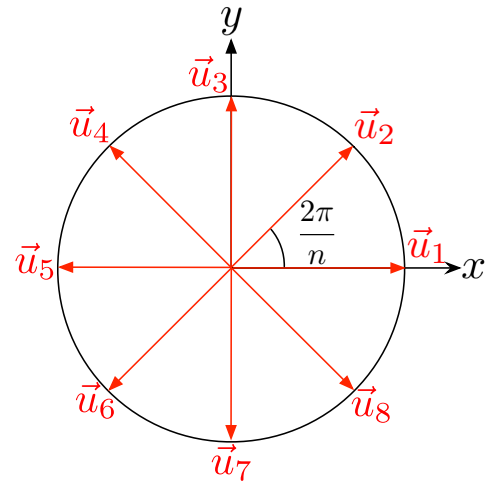

(a)

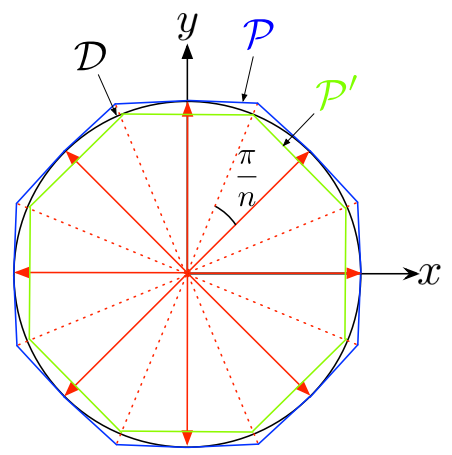

(b)

Figure 4: Linear approximation of the Euclidean norm based on directions of the Plane 
In [5], two fundamental properties are based on these directions to obtain the linearization of the Euclidean distance constraints. Let $u, v \in \mathbb{R}^{2}$. The first property allows to establish an upper bound on the Euclidean distance $\|u-v\|$ given a lower bound on the scalar products of vector $\|u-v\|$ and each direction $U_{u}$ :

$$
\left[\forall i \in \mathcal{U},\left\langle u-v \mid U_{i}\right\rangle \leq d\right] \Longrightarrow\|u-v\| \leq \frac{d}{\cos \frac{\pi}{n_{\text {directions }}}}
$$

Hence if linear constraints (12) are all satisfied, the distance between the beam center $\left(x_{b}, y_{b}\right)$ and the station $\left(X_{\text {stations }}, Y_{\text {stations }}\right)$ is lower than the beam radius. The second property gives a lower bound on the Euclidean distance $\|u-v\|$ given a lower bound on one of the scalar products of vector $\|u-v\|$ and a particular direction $U_{u}$ :

$$
\left[\exists i \in \mathcal{U},\left\langle u-v \mid U_{i}\right\rangle \geq d\right] \Longrightarrow\|u-v\| \geq d
$$

To actually linearize the Euclidean norm separations, we need another set of binary variables $\gamma_{b, b^{\prime}, u} \in\{0,1\}$ defined for all $b, b^{\prime} \in \mathcal{B}$ such that $b^{\prime}>b$ and for all $u \in \mathcal{U}$. These variables allow to rely on the second property through the constraints (13). More precisely:

- For at least one $u \in \mathcal{U}$ we must have $\gamma_{b, b^{\prime}, u}=1$. This $u$ direction will be the direction for which the projection of the vector "beam center $b$ to beam center $b$ " will be constrained to be greater than the required separation

- For $u \in \mathcal{U}$, the $\gamma_{b, b^{\prime}, u}=0$ value will be used (see constraint (14)) to relax the constraints.

This behavior with respect to the values of the $\gamma_{b, b^{\prime}, u}$ variables is ensured by constraints (14). To relax properly the separation constraint when it is necessary to do so $\left(\gamma_{b, b^{\prime}, u}=0\right)$, the coefficient $N \in \mathbb{R}^{+}$is needed. If we set $N=N_{1}+N_{2}$ where $N_{1}$ is the maximum separation distance possible between two beams and $N_{2}$ the maximum distance between two beam centers (resulting from constraint (12)),

$$
N=\underbrace{\kappa \max _{w \in \mathcal{W}} W_{w}}_{N_{1}}+\underbrace{\max _{s, s^{\prime} \in \mathcal{S}}\left\|S_{\text {coord }, s^{\prime}}-S_{\text {coord }, s}\right\|+\frac{\max _{w \in \mathcal{W}} W_{w}}{\cos \left(\frac{\pi}{n_{\text {directions }}}\right)}}_{N_{2}}
$$

then one can see that such $N$ is the lowest suitable coefficient for the aimed constraint relaxation. Indeed, for all $b, b^{\prime} \in \mathcal{B}$ and for all $u \in \mathcal{U}$, and whatever the values taken by the variables, the Cauchy-Schwarz inequality tells us that:

$$
\begin{aligned}
\left(\begin{array}{l}
x_{b^{\prime}}-x_{b} \\
y_{b^{\prime}}-y_{b}
\end{array}\right)^{T}\left(\begin{array}{l}
U_{u, x} \\
U_{u, y}
\end{array}\right) & \geq-\| \text { beam_center } b_{b^{\prime}}-\text { beam_center }_{b} \| \\
& \geq-N_{2}
\end{aligned}
$$

Besides, by definition of $N_{1}$ :

$$
\frac{1}{2}\left(\sum_{w \in \mathcal{W}} W_{w} \omega_{b, w}+\sum_{w \in \mathcal{W}} W_{w} \omega_{b^{\prime}, w}\right) \varepsilon+\lambda_{b, b^{\prime}} \leq N_{1}
$$

Therefore, the following inequality is always true:

$$
\left(\begin{array}{l}
x_{b^{\prime}}-x_{b} \\
y_{b^{\prime}}-y_{b}
\end{array}\right)^{T}\left(\begin{array}{l}
U_{u, x} \\
U_{u, y}
\end{array}\right)-\frac{1}{2}\left(\sum_{w \in \mathcal{W}} W_{w} \omega_{b, w}+\sum_{w \in \mathcal{W}} W_{w} \omega_{b^{\prime}, w}\right) \varepsilon-\lambda_{b, b^{\prime}}+N \geq 0
$$

which guarantees the relaxation of the constraint. 


\section{Upper bound for the maximum number of beams that cover at least one station}

In the linear model presented in the previous section, it is necessary to know in advance the maximum number $N_{B}$ of beams that can be used since each possible beam will have dedicated variables and constraints. In [5], the number of beams was considered as an arbitrary input of the problem. The number of beams that can be embarked on the satellite payload can be limited for mass, accommodation or cost reasons, but it can also be limited by the service area considered. In this section we propose a new upper bound on the number of beams.

If we suppose that each beam must serve at least one user ground station (otherwise some beams would be useless), then we can define for each beamwidth an admissible domain for the beam centers: all the points from which at least one station is geometrically covered. Then, both the antenna constraints and the non-overlapping constraints make it impossible to position a large number of beams on these restricted center domains.

In what follows, we propose a methodology to compute an upper bound on the maximum number $\bar{N}_{B}$ of beams that can be placed such that each beam covers at least one station with all the separation constraints satisfied. We first establish the following lemma.

Recall that $\kappa \in\left[\frac{3}{2}, \sqrt{3}\right]$ and $\varepsilon \in\left[\frac{1}{4}, \frac{\sqrt{3}}{2}\right]$ are the coefficients characterizing the angular separation for the antenna constraint and the non-overlapping constraint, respectively. Let $b, b^{\prime} \in \mathbb{R}^{2}$ be the beam centers of two distinct beams and $\omega, \omega^{\prime} \in \mathbb{R}^{+}$their beamwidths. As already discussed, the separation constraints are of the following form:

$$
\begin{gathered}
\left\|b^{\prime}-b\right\| \geq \kappa\left(\frac{\omega+\omega^{\prime}}{2}\right) \quad \text { if }\left(b, b^{\prime}\right) \text { are assigned to the same reflector antenna } \\
\left\|b^{\prime}-b\right\| \geq \varepsilon\left(\frac{\omega+\omega^{\prime}}{2}\right) \quad \text { for any }\left(b, b^{\prime}\right)
\end{gathered}
$$

Lemma 1. Considering only the smallest beamwidth yields a valid upper bound of $\bar{N}_{B}$

Proof. One can observe that the narrower the beams, the less pronounced the angular separation constraint. Consider set of beams covering at least one station and satisfying the separation constraints. Assume that at least one beam has a beamwidth that is not the smallest one. Then we can produce a new valid configuration by replacing that beam by a smaller beam that covers at least one station too. This is due to the fact that the total number of covered stations is not taken into account in the bound computation. The advantage is that with a smaller beam, the spatial impact of the separation constraint is lower, so the chance to be able to add a new beam to the coverage is higher. Concretely, it means that to find the maximum number of beams that can be placed on a given service area, one has to consider only the smallest beam size.

Now, considering only the smallest beamwidth, we compute an upper bound of $\bar{N}_{B}$ considering only the antenna constraints. The non-overlapping constraints will be addressed subsequently. The following lemma further simplifies the computation. Recall that $N_{R}$ denotes the number of reflectors.

Lemma 2. A valid upper bound of $\bar{N}_{B}$ is given by $N_{R}$ times a valid upper bound of $\bar{N}_{B}$ considering that a single reflector is available

Proof. Let $\bar{N}_{B}^{1}$ an upper bound of $\bar{N}_{B}$ considering only one reflector and suppose that $N_{R} \times \bar{N}_{B}^{1}$ is not a valid upper bound for a number of reflectors equal to $N_{R}$. Consider a solution with strictly more than $N_{R} \times \bar{N}_{B}^{1}$. We can derive from this solution a solution for one reflector with strictly more than $\bar{N}_{B}^{1}$ beams, a contradiction.

Thanks to this lemma, let us assume that we are working with only one reflector antenna. The idea is to rely on an analysis of the available and required surfaces. From the set of stations, we derive the set of possible beam centers according to the constraint to cover at least one station for each beam placed with a minimum beamwidth. This domain is delimited by the thin white polygon surrounding the punctual white user stations in Fig. 5(a). Let $\omega_{\text {min }}$ denote the minimum bandwidth. Now consider the domain $\mathcal{D}_{\text {antenna }}$ obtained by all points reachable at distance $\kappa \frac{\omega_{\min }}{2}$ from the set of possible beam centers. Domain $\mathcal{D}_{\text {antenna }}$ is represented in thick white in Fig. 5(a). 
Lemma 3. A valid upper bound of $\bar{N}_{B}$ considering only one reflector is the number of beams of diameter $\kappa \omega_{\min }$ that can be placed inside $\mathcal{D}_{\text {antenna }}$ without overlapping.

Proof. Clearly $\mathcal{D}_{\text {antenna }}$ covers the area covered by beams that cover each at least one station. Inside this area, the antenna separation constraints (21) define a non-overlapping constraints of $N_{B}$ beams of diameter $\kappa \omega_{\text {min }}$. The lemma follows.

Let now $S_{\text {antenna }}=\frac{\pi \kappa^{2} \omega_{\min }^{2}}{4}$ denote the area of one disk of diameter $\kappa \omega_{\min }$. The following theorem gives a valid upper bound of the maximum number of beams that cover each at least one station and that satisfy the antenna constraints.

Theorem 1. An upper bound of $\bar{N}_{B}$ is given by

$$
U_{\text {antenna }}=N_{R}\left\lfloor\frac{\operatorname{Area}\left(\mathcal{D}_{\text {antenna }}\right)}{S_{\text {antenna }}}\right\rfloor
$$

Proof. $\left\lfloor\frac{\operatorname{Area}\left(\mathcal{D}_{\text {antenna }}\right)}{S_{\text {antenna }}}\right\rfloor$ is an upper bound of the number of disks of diameter $\kappa \omega_{\min }$, which from Lemma 3 is an upper bound of $\bar{N}_{B}$ considering only one reflector. From Lemma 2, the theorem follows.

To build the example presented in this figure, we used $\kappa=\sqrt{3}$ and a smallest beamwidth of $\omega=0.4^{\circ}$, and we managed to place 7 beams whose corresponding disks (the transparent turquoise disks) do not intersect while the beams

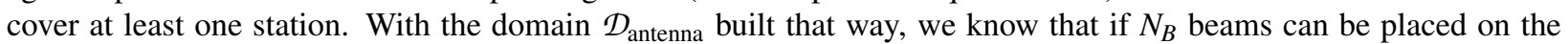
service area considered, then necessarily, $N_{B}$ times the area of one disk $S_{\text {antenna }}=\frac{\pi \kappa^{2} \omega^{2}}{4}$ is less than or equal to the area of $\mathcal{D}_{\text {antenna }}$ (because all the disks are disjoint and included in $\mathcal{D}_{\text {antenna }}$ ). What we used in practice is the contrapositive statement: In the example of Fig. 5(a), we have $\frac{\operatorname{Area}\left(\mathcal{D}_{\text {antenna }}\right)}{S_{\text {antenna }}} \simeq 10.18$ and therefore, this information would make us declare $U_{\text {antenna }}=40$ beam variables (for $N_{R}=4$ ). Looking at Fig. 5(a), it seems impossible to add 3 more beams that cover at least one station and that verify the antenna constraint, but at this point, we did not prove that it was impossible to place the 10 beams (with one antenna) announced by the surface analysis.

For the non-overlapping constraint, we can apply the exact same type of reasoning, except that the notion of reflector is irrelevant since any couple of beams is concerned by the non-overlapping constraint. From the set of centers

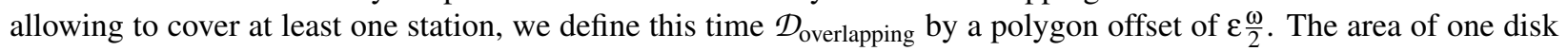
is now $S_{\text {overlapping }}=\frac{\pi \varepsilon^{2} \omega^{2}}{4}$. This time the upper bound is defined as follows:

$$
U_{\text {overlapping }}=\left\lfloor\frac{\operatorname{Area}\left(\mathcal{D}_{\text {overlapping }}\right)}{S_{\text {overlapping }}}\right\rfloor
$$

On the same service area as the one considered for the antenna constraint, Fig. 5(b) provides an example of positioning of 19 beams that respect the overlapping constraints resulting from $\varepsilon=\frac{\sqrt{3}}{2}$. The area computations lead to $\frac{\text { Area }\left(\mathcal{D}_{\text {overlapping }}\right)}{S_{\text {overlapping }}} \simeq 28.06$ and therefore, this information would make us declare $U_{\text {overlapping }}=28$ beam variables. Once again, it seems hard to add 9 more beams that cover at least one station and that do not overlap too much, but at this point it has not been proven that 28 beams could not be placed.

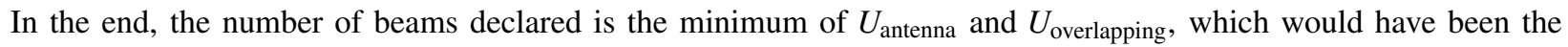
latter in the example of Fig. 5.

\section{Reduction of the problem size through k-means clustering}

The mixed-integer linear programming model MILP1 contains a significant amount of symmetries: from any solution we can build another solution of equal objective value just by permuting the indices of the beams, since they 


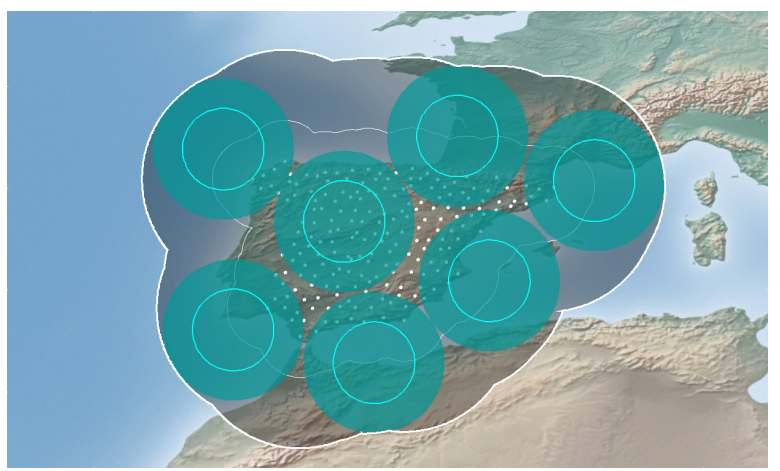

(a)

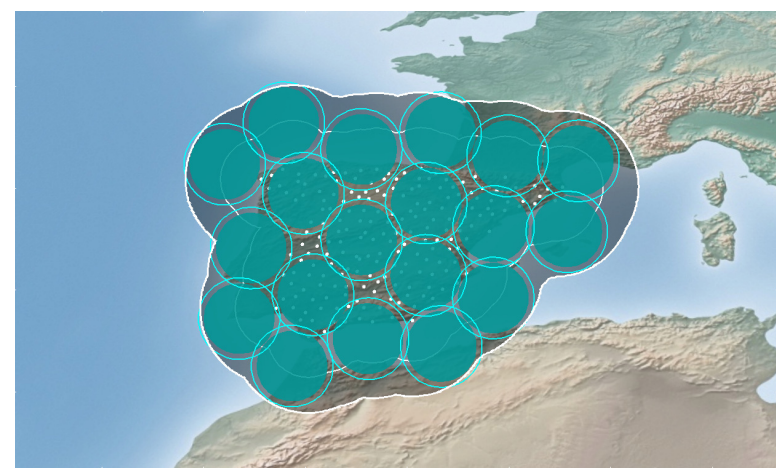

(b)

Figure 5: Surface analysis examples for computing an upper bound of the number of beams that can be placed on a given service area (a) for the antenna constraint and $\kappa=\sqrt{3}$ (b) for the non-overlapping constraints and $\varepsilon=\frac{\sqrt{3}}{2}$

can all be placed anywhere, use any size and any reflector. If each beam position was limited to a certain subset of the whole continuous set of possible beam centers implicitly present in the linear program MILP1, it would make it less easy or even impossible in some cases to generate, from a given solution, equivalent solutions just by permuting the indices: the beam center position of beam $i$ might be forbidden for beam $j$. In addition to this symmetry breaking, it would also mean that for each beam, some input user stations could not be reached from its authorized positions: less beam-station variables would be necessary. On the other hand, such limitations could result in an undesirable loss of generality for the problem solved: with inappropriate domain limitations for the beam positions and inappropriate numbers of beams per "region" of the service area, some beam layout solutions reachable with the original model could not be produced. In this section, we propose a solution based on "k-means clustering" to perform this spatial symmetry breaking. Besides, the method proposed also opens the door to several reductions of the model size. Finally, we prove that under several conditions, which we detail, there is no loss of generality with the $k$-means based mathematical model in the sense that it can produce any solution of the original model MILP1. On the other hand, we show that accepting to lose this generality can be extremely efficient for problem size reduction purposes, while suppressing only the less promising parts of the solution space regarding the optimization objective. The optimization of the number of clusters is also discussed.

In 4.1, the $k$-means clustering approach is presented, as well as the linear constraints that restrict each beam to be centered inside the polyhedron (so called Voronoï polyhedron) that corresponds to its assigned cluster. In 4.2, we explain how the clustering can help in reducing the number of binary variables linking the stations and the beams. In 4.3, we show that the presence of clusters reduces the number of directions that are relevant for the estimation of the covering of a station by a beam. In 4.4 , similar principles are used to reduce the number of binary variables that are used for beam separation constraints. The resulting mixed-integer linear programming model is then introduced in 4.5. A key point of the revised model lies in the maximum number of beams that can be assigned to a given cluster/Voronoï polyhedron. Indeed the larger this number, the larger the number of variables and constraints of the revised MILP. In 4.6, we propose methods for computing an upper bound and the exact value of this number. We also propose a heuristic to further decrease this number, possibly yielding a loss of generality in the sense that the optimal solution of the resulting MILP may be a suboptimal solution of the original MILP. In 4.7, the effect of the exact and heuristic computation of the macimum number of beams per cluster in terms of variable and constraint reduction is avaluated in fuction of the maximum number of considered clusters.

\subsection{Voronoï polyhedra and k-means clustering for disjoint beam center domains}

For a given set $\mathcal{S}=\left\{X_{1}, \cdots, X_{d}\right\}$ of $d$ real vectors of $\mathbb{R}^{n}$ and a given $k \in \mathbb{N}_{+}^{*}$, performing a $k$-means clustering 
means searching for a partition of $\mathcal{S}$ into $k$ clusters $\tilde{S}_{1}, \cdots, \tilde{S}_{k}$ that minimizes

$$
\sum_{i=1}^{k} \sum_{X \in \tilde{S}_{i}}\left\|X-\Omega_{i}\right\|^{2}
$$

where

$$
\forall i \in \llbracket 1, k \rrbracket, \Omega_{i}=\frac{1}{\operatorname{card}\left(\tilde{S}_{i}\right)} \sum_{\tilde{X} \in \tilde{S}_{i}} \tilde{X}
$$

For details on this particular clustering problem and its numerical complexity see for instance [16]. The problem is NPhard but there exist fast approximation algorithms such as the one of Lloyd [15] that we use in this paper. Clustering is naturally used to reduce the size of the input data of large-scale optimization problems as a means to aggregate the data. For example in [9], $k$-means clustering is used to reduce the number of days for long term optimization of a district energy system. Clustering and, in particular, $k$-means clustering has been widely used for facility location problems. In [13], $k$-means clustering is used as a heuristic to solve the (discrete) capacitated facility location problem. In [17], a continuous capacitated facility location problem is considered. The problem is closer to our problem as the facilities (that could be identified with the beams) must be positioned with continuous coordinates in the plane so as to serve a set of users. However, the problem is considerably simpler as the non linearities appear only in the objective, which is to minimize the total euclidean distance from the users to the assigned facilities. A heuristic alternates a phase facilities location with a phase of assignment of users to facilities using $k$-mean clustering. In [25], the authors compare, for the same continuous facility location problemm, $k$-mean heuristics to a MILP formulation based on euclidean distance linearization. However, they did not use $k$-mean clustering to reduce the size of the resulting MILP.

Note that one property that should be verified by any vector clustering produced with the objective of equation (24) is that each vector of $\mathcal{S}$ is associated to the cluster with the nearest mean $\Omega_{i}$, otherwise there would exist obvious transfers of points from one cluster to another that would directly improve the optimization objective. In particular, this is indeed verified with Lloyd's algorithm when it converges (most widely used $k$-means clustering heuristic [15]). Note that this algorithm is the one we decided to consider and use in this work to form clusters of user stations. Therefore, let us consider a partition in $k$ clusters $\tilde{S}_{1}, \cdots, \tilde{S}_{k}$ built this way and let $(i, j) \in \llbracket 1, k \rrbracket^{2}(i \neq j)$. We introduce the following notation:

$$
N_{i j}=\left(\begin{array}{c}
\Omega_{j 1}-\Omega_{i 1} \\
\vdots \\
\Omega_{j n}-\Omega_{i n}
\end{array}\right) \in \mathbb{R}^{n} \quad \Lambda_{i j}=\frac{1}{2} \sum_{h=1}^{n}\left(\Omega_{j h}^{2}-\Omega_{i h}^{2}\right) \in \mathbb{R} \quad \mathcal{H}_{i j}=\left\{X \in \mathbb{R}^{n} /\left\langle X \mid N_{i j}\right\rangle \leq \Lambda_{i j}\right\}
$$

Let us now consider $X \in \tilde{S}_{i}$, its closest mean being $\Omega_{i}$ since we assume that Lloyd's algorithm has converged, we have necessarily

$$
\left\|X-\Omega_{i}\right\|^{2} \leq\left\|X-\Omega_{j}\right\|^{2} \quad \forall j \in \llbracket 1, k \rrbracket
$$

That is

$$
\underbrace{\sum_{h=1}^{n}\left(\frac{\Omega_{i h}^{2}}{2}-\frac{\Omega_{j h}^{2}}{2}-X_{h} \Omega_{i h}+X_{h} \Omega_{j h}\right)}_{=\left\langle X \mid N_{i j}\right\rangle-\Lambda_{i j}} \leq 0 \quad \forall j \in \llbracket 1, k \rrbracket
$$

This set of $k$ linear constraints can be used to enforce each beam center to belong to its assigned cluster. Therefore, $X \in \mathcal{H}_{i j}$. Since it is true for all $(i, j) \in \llbracket 1, k \rrbracket^{2}$ such that $i \neq j$, it means that equivalently to (28), we can write:

$$
\forall i \in \llbracket 1, k \rrbracket, \forall X \in \tilde{S}_{i}, \quad X \in \bigcap_{\substack{j \in \llbracket 1, k \rrbracket \\ j \neq i}} \mathcal{H}_{i j} \underbrace{=}_{\text {notation }} \mathcal{V}_{i}
$$

In the $k$-means clustering terminology, the $\mathcal{V}_{i}$ sets (clusters) are called Voronoï cells. We make sure that all the $\mathcal{V}_{i}$ sets are bounded in $\mathbb{R}^{n}$ by adding the following half-spaces in their definition: $\forall i \in \llbracket 1, k \rrbracket, \forall X \in \mathcal{V}_{i}, \forall h \in \llbracket 1, n \rrbracket, X_{h} \geq$ $l_{h, \text { min }}$ and $X_{h} \leq l_{h, \text { max }}$, the $l_{1, \text { min }}, \cdots, l_{n, \text { min }}$ and $l_{1, \max }, \cdots, l_{n, \max }$ coefficients being chosen as to have large enough 
$l_{h, \max }-l_{h, \text { min }}$ values to entirely contain the subset of $\mathbb{R}^{n}$ of interest. The novel approach we propose in this paper is to use the Voronoï cells derived from a $k$-means clustering of the user stations (vectors of $\mathbb{R}^{2}$ representing projected true view angles) as admissible domains for the continuous Euclidean point variables of our mixed-integer linear programming model of beam layout optimization. This is perfectly possible since these cells are intersections of half-spaces, which corresponds to the most natural constraints in linear programming. This allows to reach a model with both reduced size and less symmetries, while keeping the possibility to have a model as general as the original one (1-11) under certain conditions detailed below. We also discuss the possibility to give up this generality to reduce even more the problem size, this heuristic approach leading to better practical results. Most importantly, let us insist on the fact that this exploitation of the Voronoï cells appears as a method that could be transposed to other applications than only the beam layout optimization, on other facility location problems for instance.

In practice, after the $k$-means clustering of the user stations, each cluster $i$ is assigned a maximum number of beams $\mathcal{N} i \geq 1$, according to a certain strategy depending on whether or not the generality of the model needs to be preserved (discussed in a paragraph below). Each beam $b \in \mathcal{B}$ is now characterized by its cluster index $c_{b} \in \llbracket 1, k \rrbracket$. Then, each beam $b \in \mathcal{B}$ is constrained to belong to $\mathcal{V}_{c_{b}}$, which is done by the conjunction of $k-1$ constraints as shown in Fig. 6. Each of the sub-figures (a) ... (h) corresponds to the adjunction of one of the constraints (28) so that the feasible region converges toward the Voronoï cell.

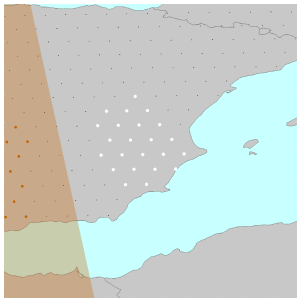

(a)

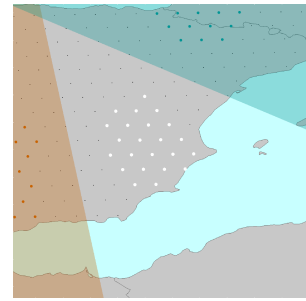

(b)

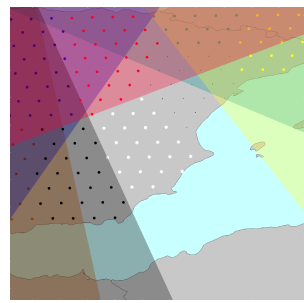

(f)

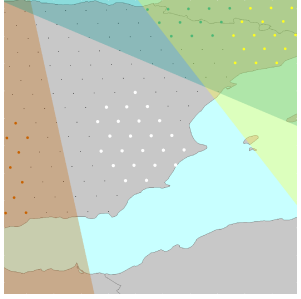

(c)

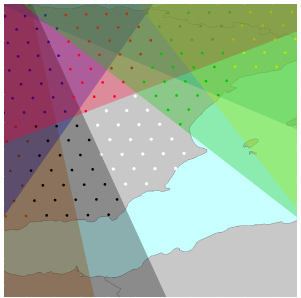

(g)

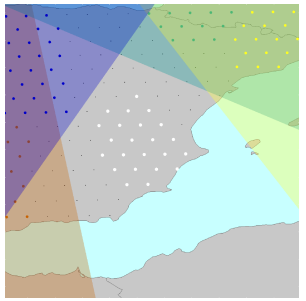

(d)

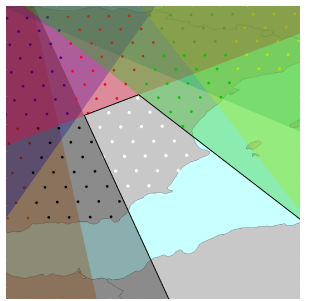

(h)

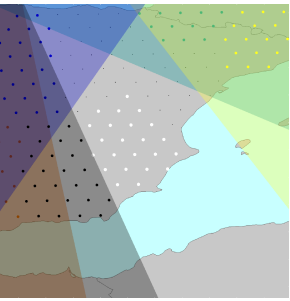

(e)

Figure 6: Restriction of a beam of a given cluster to its corresponding Voronoï cells: intersection of $k-1$ half-spaces, one for each cluster other than the cluster considered

\subsection{Reduction of the number station-beam variables}

One direct consequence of this restriction of the domain centers to the Voronoï polyhedra is that, for a given beam, only the stations in or close to the Voronoï cell it belongs to are reachable: less $\alpha_{s, b}$ variables are necessary than in the model (1-11). More precisely, for each cluster $i$, the set of reachable stations $\mathcal{S}_{i} \subset \mathcal{S}$ is directly linked the maximum possible beamwidth:

$$
S_{i}=\left\{s \in \mathcal{S} / \min _{X \in \mathcal{V}_{i}}|| S_{\text {coord }, s}-X \| \leq \frac{1}{2} \frac{\max _{w \in \mathcal{W}} W_{w}}{\cos \left(\frac{\pi}{n_{\text {directions }}}\right)}\right\}
$$

An example is provided in Fig. 7. Part (a) of Fig. 7 shows in red the stations assigned to a given cluster, while part (b) of Fig. 7 displays in red the set $S_{i}$ of reachable stations. Some more useful notation should be introduced to match 
this reduction of $\alpha_{s, b}$ variables: for each station $s \in \mathcal{S}, \mathcal{B}_{s} \subset \mathcal{B}$ denotes the set of beams that can cover $s$ and for each beams $b \in \mathcal{B}, \hat{S}_{b} \subset \mathcal{S}$ denotes the set of stations that can be covered by $b$.

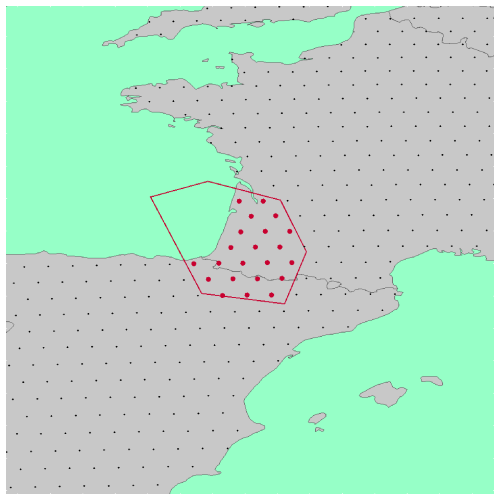

(a)

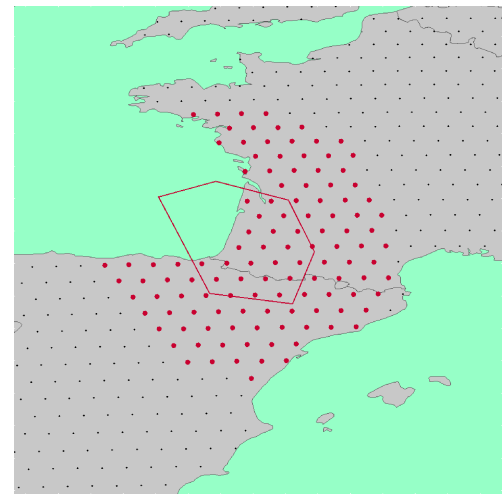

(b)

Figure 7: For a given cluster, example of contained stations (a) and of reachable stations (b)

\subsection{Reduction of the number of station-beam-direction constraints}

Let $i$ be one of the station clusters and let $s \in \mathcal{S}_{i}$ such that $S_{\text {coord,s }} \notin \mathcal{V}_{i}$. Since $\mathcal{V}_{i}$ is obviously convex (so is the singleton $\left\{S_{\text {coord,s }}\right\} \in \mathbb{R}^{2}$ ), the hyperplane separation theorem tells us that there exists a straight line separating strictly $\mathcal{V}_{i}$ and $\left\{S_{\text {coord,s }}\right\}$. The resulting property here is that the set of possible angles $\left(S_{\text {coord,s }} \mid X\right)$ when $X \in \mathcal{V}_{i}$ is in an interval of length less than or equal to $\pi$.

By contradiction, it can be shown that the extreme directions that define this angular range are to be found among the $V-S_{\text {coord,s }} \in \mathbb{R}^{2}$ directions, where $V$ is an extreme point of the convex polytope $\mathcal{V}_{i}$. In Fig. 8, the only relevant directions for the considered station out of the Voronoï cell are represented : they lie in the larger range defined by the segments connecting the station to the extreme points of the polytope. For a certain beam $b \in \mathcal{B}$ placed at $X \in \mathcal{V}_{i}$, the interesting property we can derive from this result is that, among the $n_{\text {directions }}$ discretized directions defined according the linearization process of [5], we can be sure that the "closest direction" to the vector $X-S_{\text {coord, } s}$ is one of the discretized directions contained in this angular range, this subset of directions being denoted by $\mathcal{U}_{s, i}^{\prime} \subset \mathcal{U}$. It is sufficient to consider only these directions in constraints (12): the number of constraints is reduced. Note that for $s \in S_{i}$ such that $S_{\text {coord,s }} \in \mathcal{V}_{i}$, we will set $\mathcal{U}_{s, i}^{\prime}=\mathcal{U}$ since this simplification is not applicable.

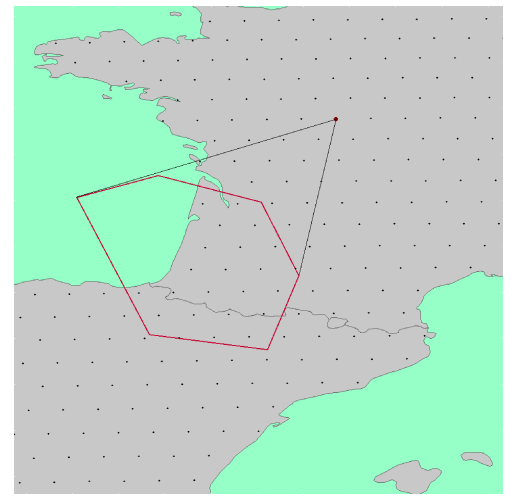

Figure 8: Angular range for a reachable station out of the Voronoï cell 


\subsection{Reduction of the beam-beam variables}

Regarding the couples of beams and the antenna constraint that forces them to be sufficiently separated if they use the same satellite reflector, one major advantage of the clustering is that we have the guarantee that some couples of beams can never be closer than the threshold distance under which they must use different reflectors (which by transitivity guarantees that the beam cannot overlap too much since the overlapping threshold is a shorter distance than the antenna separation angle). Indeed, for $b$ belonging to a certain cluster $i$ and $b^{\prime}$ belonging to cluster $j$ different than $i$, if

$$
\min _{X \in \mathcal{V}_{i}, Y \in \mathcal{V}_{j}}|| X-Y \| \geq \max _{w \in \mathcal{W}} W_{w}
$$

then we are sure that $b$ and $b^{\prime}$ will always be sufficiently separated and do not need specific $\beta_{b, b^{\prime}}$ and $\lambda_{b, b^{\prime}}$ variables. On the other hand, when

$$
\min _{X \in \mathcal{V}_{i}, Y \in \mathcal{V}_{j}} \mid\|X-Y\|<\kappa \max _{w \in \mathcal{W}} W_{w}
$$

we both need $\beta_{b, b^{\prime}}$ and $\gamma_{b, b^{\prime}, u}$ variables, but just like in the previous paragraph with the out-of-cluster stations, the number of $\gamma_{b, b^{\prime}, u}$ variables and the corresponding constraints (14) can be reduced by considering only the relevant discretized directions $\mathcal{U}_{b, b^{\prime}}^{\prime} \subset \mathcal{U}$ for $\left(b, b^{\prime}\right)$. Indeed, since the different clusters are either disjoint or share at most one edge, we also have the guarantee that this range of angles of amplitude less than or equal to $\pi$ also exists for the beam-center to beam-center vectors. Fig. 9 illustrates this principle. Part (a) of Fig. 9 displays the case of two clusters sharing an edge, in which case the angular range for the relevant directions is equal to $\pi$. Part (b) displays the case of disjoint clusters for which the angular range if the relevant directions (represented by the segments joining the clusters) is strictly lower then $\pi$. Note though that none of these simplifications can be applied to a couple of beams both belonging to the same cluster. In the end, we will denote by $\mathbf{B} \subset \mathcal{B}^{2}$ the set of beam couples $\left(b, b^{\prime}\right)$ (with $b>b^{\prime}$ to avoid variable and constraint redundancies) for which we do need $\beta_{b, b^{\prime}}$ and $\gamma_{b, b^{\prime}, u}$ variables to express the antenna constraint.

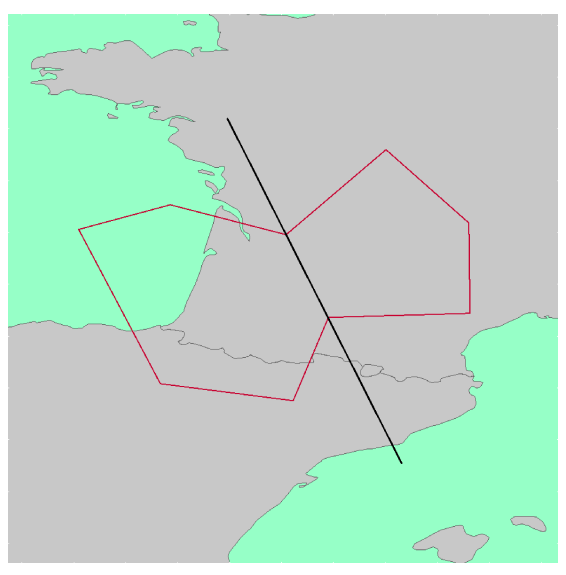

(a)

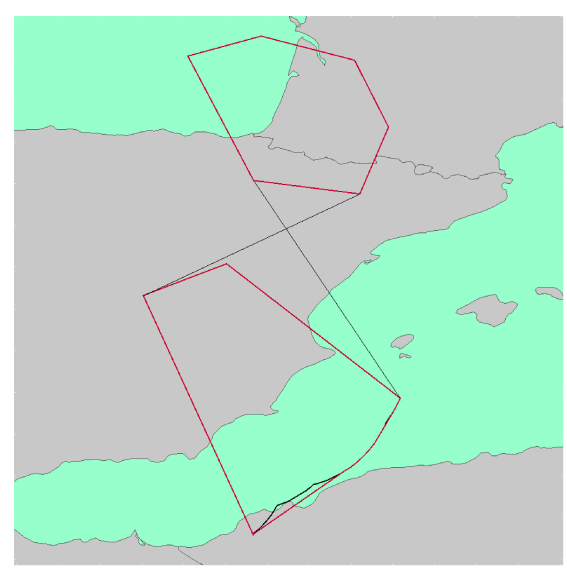

(b)

Figure 9: (a) Edge-sharing clusters angular range (equal to $\pi$ ) (b) Disjoint clusters angular range (lower than $\pi$ )

\subsection{Revised mixed-integer linear programming model}

In addition to the reduced sets of variables and constraints and to the constraint to belong to the Voronoï cells, the way the $N$ and $M_{s}$ values, respectively defined in (14) and (4), has changed in the new model. For such relaxation coefficients, the lower the better to avoid detrimental numerical behaviors, and in the clustering-based model, these values can be tightened compared to those of the original model MILP1. Concerning the $N$ coefficient, it is now defined for all $i, j \in \llbracket 1, k \rrbracket$ and set to $N_{i, j}=\kappa^{m a x} \max _{w \in \mathcal{W}} W_{w}+\max _{X \in \mathcal{V}_{i}, Y \in \mathcal{V}_{j}}\|X-Y\|$ for the same reasons as before. 
In the case of $M_{s}$, this value is now defined for each cluster $i \in \llbracket 1, k \rrbracket$ and for each station $s \in S_{i}$, and is set to $M_{i, s}=\max _{s^{\prime} \in \mathcal{S}_{i}}|| S_{\text {coord }, s^{\prime}}-S_{\text {coord }, s} \|$ also for the same reasons as before. The constraint (34) is directly based on equation (28) and defines the Vorono cells. Finally, the constraint (46) to have a total of at most $n_{\max }$ beams is also added. In the end, we reach model (33-46), denoted as MILP2.

$$
\operatorname{Maximize} \sum_{s \in \mathcal{S}} \sum_{b \in \mathcal{B}_{s}} T_{s} \alpha_{s, b}
$$

under the following constraints

$$
\begin{aligned}
& \forall b \in \mathcal{B}, \forall i \in \llbracket 1, k \rrbracket \text { such that } i \neq c_{b}, \quad\left(\Omega_{i 1}-\Omega_{c_{b} 1}\right) x_{b}+\left(\Omega_{i 2}-\Omega_{c_{b} 2}\right) y_{b} \leq \Lambda_{c_{b} i} \\
& \forall s \in \mathcal{S}, \quad \sum_{b \in \mathcal{B}_{s}} \alpha_{s, b} \leq 1 \\
& \forall b \in \mathcal{B}, \quad \sum_{w \in \mathcal{W}} \omega_{b, w}=1 \\
& \forall s \in \mathcal{S}, \forall b \in \mathcal{B}_{s}, \forall u \in \mathcal{U}_{s, c_{b}}^{\prime}, \quad\left(\begin{array}{c}
x_{b}-X_{\text {stations }, s} \\
y_{b}-Y_{\text {stations }, s}
\end{array}\right)^{T}\left(\begin{array}{c}
U_{u, x} \\
U_{u, y}
\end{array}\right) \leq \frac{1}{2} \sum_{w \in \mathcal{W}} W_{w} \omega_{b, w}+\left(1-\alpha_{s, b}\right) M_{c_{b}, s} \\
& \forall b \in \mathcal{B}, \quad \sum_{r \in \mathcal{R}} \rho_{b, r}=1 \\
& \forall\left(b, b^{\prime}\right) \in \mathbf{B}, \quad \lambda_{b, b^{\prime}} \geq \frac{\kappa-\varepsilon}{2} \sum_{w \in \mathcal{W}} W_{w}\left(\omega_{b, w}+\omega_{b^{\prime}, w}\right)-(\kappa-\varepsilon) \max _{w \in \mathcal{W}} W_{w} \beta_{b, b^{\prime}} \\
& \forall\left(b, b^{\prime}\right) \in \mathbf{B}, \quad \sum_{u \in \mathcal{U}^{\prime}, b^{\prime}} \gamma_{b, b^{\prime}, u} \geq a_{b}+a_{b^{\prime}}-1 \\
& \forall\left(b, b^{\prime}\right) \in \mathbf{B}, \forall u \in \mathcal{U}_{b, b^{\prime}}^{\prime}, \\
& \left(\begin{array}{l}
x_{b^{\prime}}-x_{b} \\
y_{b^{\prime}}-y_{b}
\end{array}\right)^{T}\left(\begin{array}{l}
U_{u, x} \\
U_{u, y}
\end{array}\right) \geq \frac{1}{2}\left(\sum_{w \in \mathcal{W}} W_{w} \omega_{b, w}+\sum_{w \in \mathcal{W}} W_{w} \omega_{b^{\prime}, w}\right) \varepsilon+\lambda_{b, b^{\prime}}-N_{c_{b}, c_{b^{\prime}}}\left(1-\gamma_{b, b^{\prime}, u}\right) \\
& \forall\left(b, b^{\prime}\right) \in \mathbf{B}, \forall r \in \mathcal{R}, \quad \beta_{b, b^{\prime}}+\rho_{b, r}+\rho_{b^{\prime}, r} \leq 2+\left(1-a_{b}\right)+\left(1-a_{b^{\prime}}\right) \\
& \forall b \in \mathcal{B}, \quad \sum_{s \in \hat{\mathcal{S}}_{b}} \alpha_{s, b} \leq \operatorname{card}\left(\hat{\mathcal{S}}_{b}\right) a_{b} \\
& \forall b \in \mathcal{B}, \quad \sum_{s \in \hat{\mathcal{S}}_{b}} T_{s} \alpha_{s, b} \leq \sum_{w \in \mathcal{W}} \Gamma_{w} \omega_{b, w} \\
& \forall b \in \mathcal{B}, \quad \sum_{s \in \hat{\mathcal{S}}_{b}} \alpha_{s, b} \geq N_{\min } a_{b} \\
& \sum_{b \in \mathcal{B}} a_{b} \leq n_{\max }
\end{aligned}
$$

Variables: $\alpha_{s, b}, \omega_{b, w}, \rho_{b, r}, a_{b}, \beta_{b, b^{\prime}}, \gamma_{b, b^{\prime}, u} \in\{0,1\}$ and $x_{b}, y_{b} \in \mathbb{R}$ and $\lambda_{b, b^{\prime}} \in \mathbb{R}^{+}$

With the $M_{i, s}$ values properly defined, the different constraints that impact the beam center domains are all known. As a direct complement of Fig. 6, Fig. 10 finishes to detail how the final beam center domains (denoted by $\mathcal{V}_{i}^{\prime}$ for each cluster $i$ ) are reached thanks to the constraints (37) and (45). In practice, only the boundary clusters use actively the "at least one station" constraint (which dominates the constraints (37) thanks to our choice of $M_{i, s}$ values), the Voronoï cells constraints prevailing anywhere else. In the end, what we reach on the whole service area is visible in Fig. 11. 


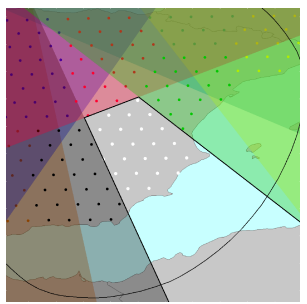

(a)

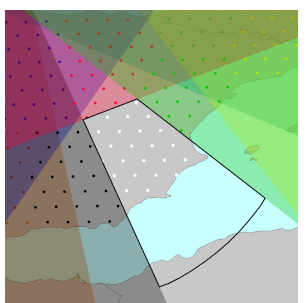

(b)

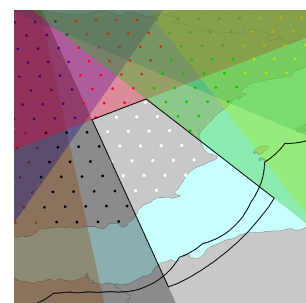

(c)

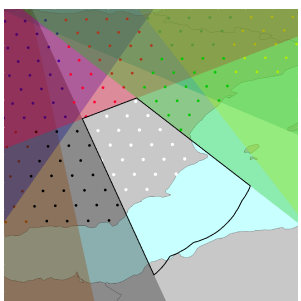

(d)

Figure 10: Domain center limitations resulting from constraints (37) on figures (a) and (b), and those resulting from constraints (45) on figure (c) and (d)

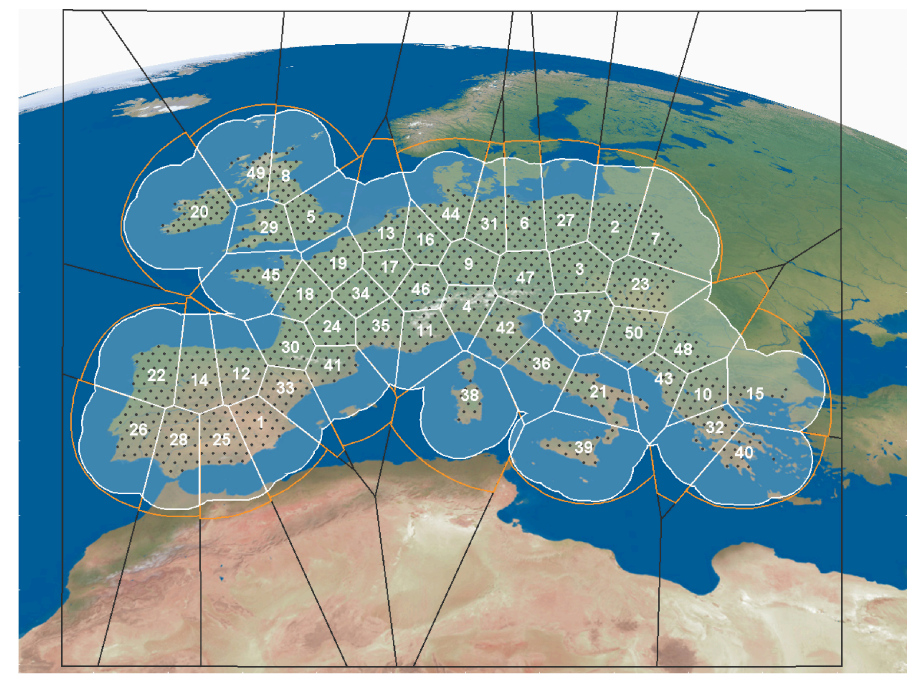

Figure 11: $k$-means clustering of the service area $(k=50)$ and admissible domains for the centers: Voronoï cells in black, domains resulting from constraint (37) in orange, and the one resulting from the constraints (45) in white

\subsection{Maximum number of beams per cluster: general versus heuristic computation}

It is obvious that any solution produced by the clustering-based model of MILP2 corresponds to a solution of the model MILP1 as the latter can be seen as a relaxation of the former. On the other hand, a solution issued by the original model might not have a corresponding solution in the clustering-based model if, for instance, the solution includes $m+1$ beams on a beam center zone exclusively accessible by a cluster $i$ for which the maximum number of beams $\mathcal{N}_{i}$ has been set to a value less than or equal to $m$. One way to make sure the clustering-based model stays general is to set for each cluster $i$ the maximum number of allowed beams in this cluster $\mathcal{N}_{i}$ to the maximum number of beams than can be actually placed on the domain $\mathcal{V}_{i}^{\prime}$, or at least to a close upper bound of this number. To estimate such an upper bound, the first step of the process we applied here consists in relying on the surface analysis presented in 3, this helps reach a first $\mathcal{N}_{i \text {,temporary }}$ Then, we propose to improve this upper bound with a MILP whose objective is to determine exactly the maximum number of beams that can be placed on cluster $i$ given only the antenna and non-overlapping separation constraints. To do so, having a model that includes all the user stations related variables and constraints is not necessary, as long as the center domains are correctly given as input. In other words, having a polytope representation $\mathcal{V}_{i, \text { polyg }}^{\prime}=\left\{X \in \mathbb{R}^{2} / \mathbf{A}_{i} X \leq B_{i}\right\}$ (with $m_{i}>0, \mathbf{A}_{i} \in \mathbb{R}^{m_{i} \times 2}$ and $B_{i} \in \mathbb{R}^{m_{i}}$ ) of $\mathcal{V}_{i}^{\prime}$ allows to reach a simple MILP, derived from MILP1, that maximizes this time the number of active beams on cluster $i$. Note that in some cases $\mathcal{V}_{i}^{\prime}$ is already naturally a polygon, only some of them are non-polygonal sets, in that case we chose to approximate them by an enclosing convex polygon of $m_{i}$ sides, meaning that we ensure $\mathcal{V}_{i}^{\prime} \subset \mathcal{V}_{i, \text { polyg }}^{\prime}$. The MILP we 
reach is given by equations (47-53) with an input maximum number of beams given by $N_{B}=\mathcal{N}_{i \text {,temporary }}$ and only one possible beamwidth: the smallest $\left(W_{\min } \in \mathbb{R}^{+}\right.$), for less important separation constraints in order to be able to place more beams. This MILP is denoted MILP2'.

$$
\begin{gathered}
\text { Maximize } \sum_{b \in \mathcal{B}} a_{b} \\
\text { under the following constraints } \\
\forall b \in \mathcal{B}, \quad \mathbf{A}_{i}\left(\begin{array}{l}
x_{b} \\
y_{b}
\end{array}\right) \leq B_{i} \\
\forall b \in \mathcal{B}, \quad \sum_{r \in \mathcal{R}} \rho_{b, r}=1 \\
\forall b, b^{\prime} \in \mathcal{B} \text { such that } b \neq b^{\prime}, \quad \lambda_{b, b^{\prime}}+(\kappa-\varepsilon) W_{\min } \beta_{b, b^{\prime}} \geq(\kappa-\varepsilon) W_{\text {min }} \\
\forall b, b^{\prime} \in \mathcal{B} \text { such that } b \neq b^{\prime}, \quad \sum_{u \in \mathcal{U}} \gamma_{b, b^{\prime}, u} \geq 1 \\
\forall b, b^{\prime} \in \mathcal{B} \text { such that } b \neq b^{\prime}, \forall u \in \mathcal{U}, \\
\forall b, b^{\prime} \in \mathcal{B} \text { such that } b \neq b^{\prime}, \forall r \in \mathcal{R}, \quad \beta_{b, b^{\prime}}+\rho_{b, r}+\rho_{b^{\prime}, r} \leq 2+\left(1-a_{b}\right)+\left(1-a_{b^{\prime}}\right) \\
\left.y_{b^{\prime}}-y_{b}\right)^{T}\left(\begin{array}{l}
U_{u, x} \\
U_{u, y}
\end{array}\right) \geq \varepsilon W_{\min }+\lambda_{b, b^{\prime}}-N_{i, i}\left(1-\gamma_{b, b^{\prime}, u}\right)
\end{gathered}
$$

In practice, MILP2' is run for a certain amount of time for each cluster, when optimality is reached and proved, $\mathcal{N}_{i}$ is set to the optimum of the MILP, otherwise $\mathcal{N}_{i}$ stays equal to $\mathcal{N}_{i \text {,temporary }}$ obtained beforehand with a surface analysis. With this strategy of $\mathcal{N}_{i}$ definition, we guarantee the generality of the clustering based model.

Since any pair of beams of the same cluster cannot take benefit from the reduction size principles presented in previous sections, it is interesting to further decrease the $\mathcal{N}_{i}$ values even if the generality is lost. The heuristic we propose here for such a choice exploits $n_{\max }$, the relative area of the cluster relatively to the original model center domain, and the extremal beamwidths that help define a "maximum relative density of beams" coefficient for the clusters :

$$
\mathcal{N}_{i}=\operatorname{ceil}\left(n_{\max }\left(\frac{\max _{w \in \mathcal{W}} W_{w}}{\min _{w \in \mathcal{W}} W_{w}}\right)^{2} \frac{\operatorname{Area}\left(\mathcal{V}_{i}\right)}{\mathcal{A}^{\prime}}\right)
$$

In practice, this definition assumes that we do not want to favor a given cluster more than another in terms of number of beams: the overall number of available beams is balanced over all the clusters, with a margin that allows to deal with the more demanding clusters (in terms of user station traffic requests) that can use more beams and therefore use multiple small beams.

\subsection{Impact of the number of clusters on variable and constraint reduction}

In this section, we evaluate the two strategies of definition of the maximum number of beams in each cluster in the clustering-based model for a varying number of clusters in terms of resulting number of variables and number of constraints. We illustrate the behavior of the two strategies on an instance here composed of 200 user stations, 2 beamwidths, 4 reflector, 12 discretized directions, $\kappa=\sqrt{3}$, and $n_{\max }=50$. Fig. 12 gives the results of the two approaches in terms number of variables (part a) and constraints (part b), in function of the number of clusters. In both cases, the clustering allows globally to decrease the number of variables and constraints. However, the heuristic process (in blue) allows to decrease the number of variables and constraints significantly below the case where no clustering is applied (in black) while the generality-preserving method (in red) fails in bringing a variable and constraint reduction, although it stills ensures symmetry breaking as each beam is assigned to a single cluster. This is due to the fact that the more beams in the clusters, the less efficient the model size reduction process. 


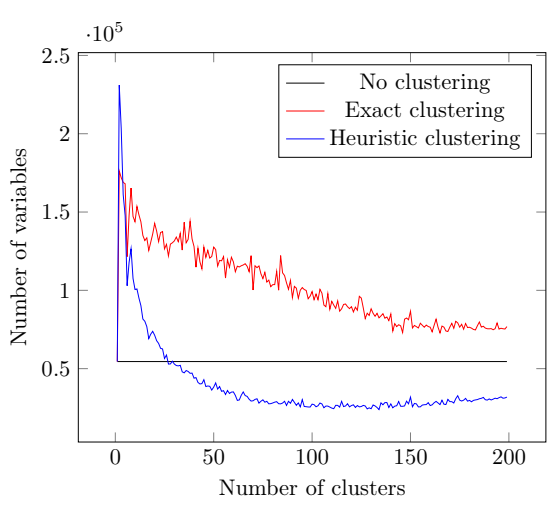

(a)

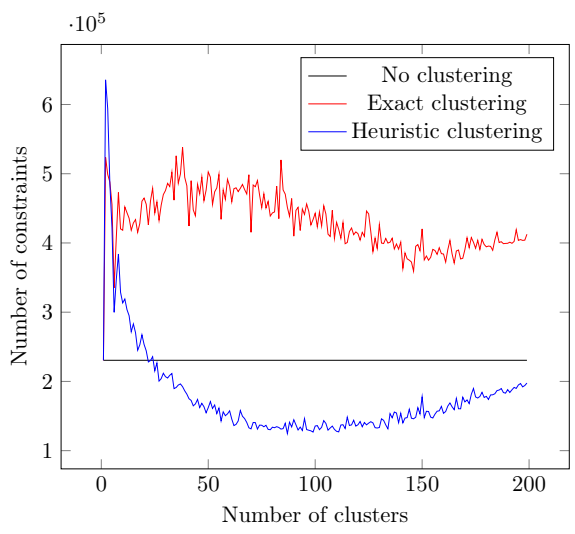

(b)

Figure 12: Evolution with respect to the number of clusters of (a) The number of variables of the revised model (b) The number of constraints of the revised model

\section{Computational experiments}

The goal of the campaign of tests we carry out is to assess the interest of our $k$-means clustering based strategy of MILP formulation improvement. To do so, we benchmarked three different algorithmic solutions:

- The raw linearized MILP model that does not rely on any reduction size principle (MILP1)

- The $k$-means clustering based model with an exact preservation of the problem generality (MILP2)

- The $k$-means clustering based model with a heuristically defined number of beams per cluster (MILP3)

\subsection{Realistic instances generation}

One necessary step for assessing properly the quality of these algorithmic solutions was to automate the definition of realistic instances, to be able to perform experimentations on a significant number of cases. In other words, we needed a process to define realistic sets of input data that entirely define the instances of the problem to be solved. In the particular case of the beam layout problem, let us remind that the parameters characterizing a beam layout instance are the following:

1) A coverage area on the surface of the Earth defined by a set of user stations

2) The spatial density of the user stations

3) Their individual traffic demands (defining a certain demand heterogeneity)

4) Maximum traffic load per beam constraints, variable in function of the beamwidth

5) A number of available reflectors

6) An angular separation rule for two beams transmitted by the same reflector

7) An overlapping rule for any two beams

8) A finite number of possible beamwidths

9) A minimum number of stations to cover per active beam 
The approach we adopted to generate our series of instances has been to set all these input parameters but the first three of this list: several grids of stations of different size and density have been generated, and for each grid, the traffic demand of each user station is randomly generated according to different heterogeneity objectives and in accordance with the actual applications encountered by Airbus Defense and Space. For these traffic demand distributions to be realistic, it is necessary to:

(i) Control the heteregeneity of the demand to be able to make it vary into a realistic range.

(ii) Avoid spatially erratic distributions: the demand should vary smoothly thus clearly defining dense and less dense zones similar to the ones that could be found by analyzing the population density

The need $(i)$ is handled by a proper tuning of the standard deviation of a normal distribution, which is the probalistic law we chose to generate initial traffic demand values. An example of the type of map we obtain that way is given in Fig. 13(a) where the traffic demand of the user stations is expressed in Megabits per second. Then, (ii) requires to treat afterwards these normally distributed values with a spatial Gaussian smoothing, which comes down to convolving these user station demands with a 2D Gaussian function (defined on the $\Theta_{x} \times \Theta_{y}$ plane). In Fig. 13, the traffic demand map (a) is transformed into (b) after this smoothing procedure.

On the maximum traffic load per beam, we needed a process to define realistic thresholds for any traffic demand map generated randomly. From an available series of realistic traffic demand maps, the idea was to define a threshold definition rule through an analysis of the traffic coverable by the smallest beam size from all the possible beam center positions. We then compared the traffic limit known for that size to the distribution of coverable traffic per beam center position: it lead to the rule (that we used throughout all our experiments) that from $\approx 95 \%$ of the possible beam center positions the coverable traffic of a beam of the smallest beamwidth is less than the traffic load limit. In practice, for each traffic map generated randomly, all the beam center positions are analyzed and the threshold for the smallest beam is set to the reachable traffic value corresponding to $95 \%$ in the cumulative distribution function of the traffic reachable per position. Then, the thresholds for the larger beamwidths are set according to a certain decreasing rule from the smallest beamwidth threshold (the larger beams cannot handle as much traffic as the small beams since they benefit from lower spectral efficiencies).

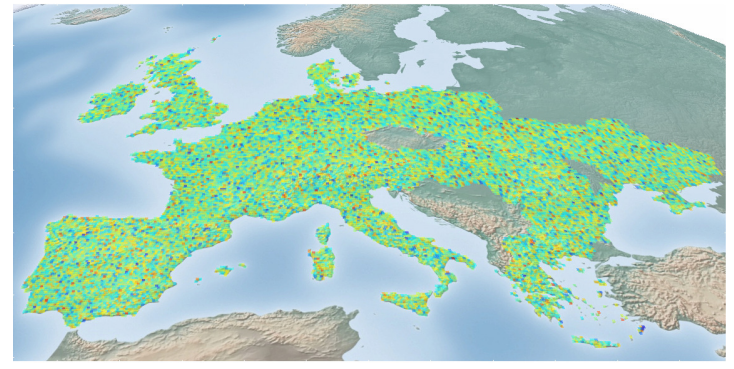

(a)

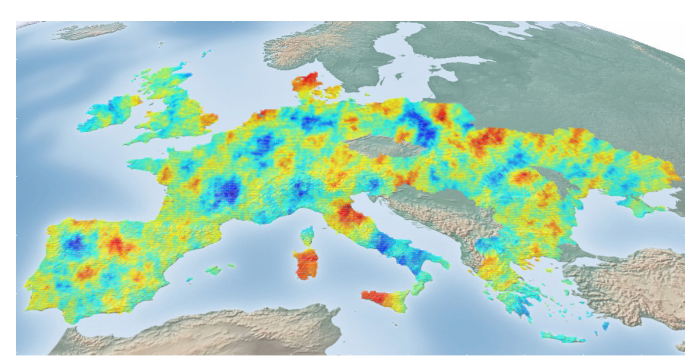

(b)

Figure 13: (a) Normal distribution before Gaussian blur (b) Distribution after Gaussian blur

\subsection{The different types of instances tested}

In this experimental context, we wanted to assess the sensitivity of the different methods to different families of instances. Two characteristics guided the way we built our campaign of tests:

- The size of the service area to be covered

- The heterogeneity of the traffic demand

These two parameters have been tuned in turn to generate five distinct sets of instances $H_{1}, \cdots, H_{5}$ characterized by decreasing traffic demand heterogeneity on the service zone, the instances in each of these sets being of growing service 
area size. More precisely the standard deviation of the normal distribution used to assign traffic demands to ground stations is decreasing for $H_{1}, \ldots, H_{5}$. In Fig. 14(a) and Fig. 14(b), we represented the two extreme configurations in terms of heterogeneity of traffic demand: $H_{1}$ with high heterogeneity (a), $H_{5}$ with low heterogeneity (b). Then, in Fig. 14(c) and Fig. 14(d), the growing size of the service zone in the instances of a given heterogeneity level is illustrated : it is once again a representation of extreme cases with the smallest and largest service zones of a given heterogeneity category.

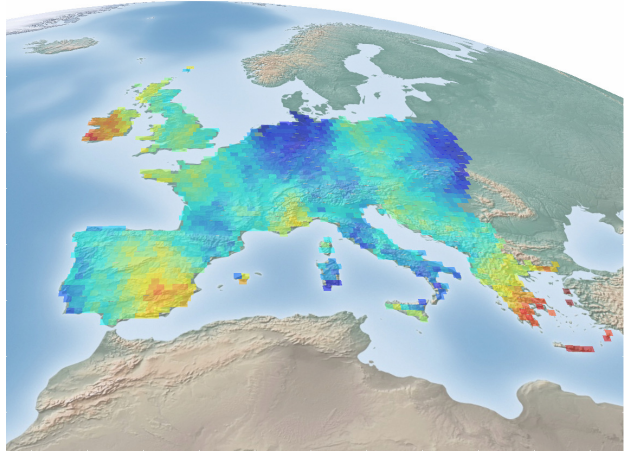

(a)

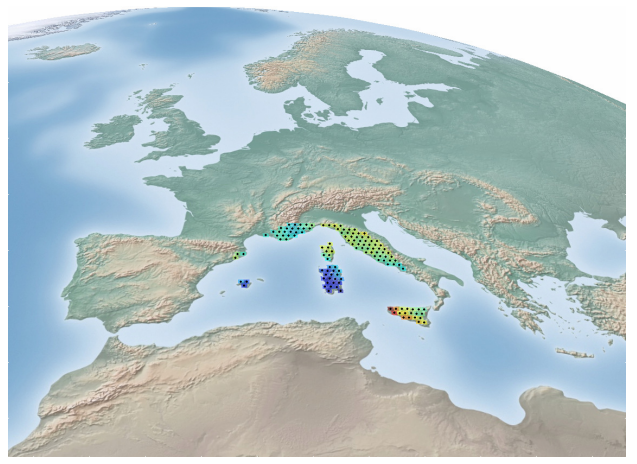

(c)

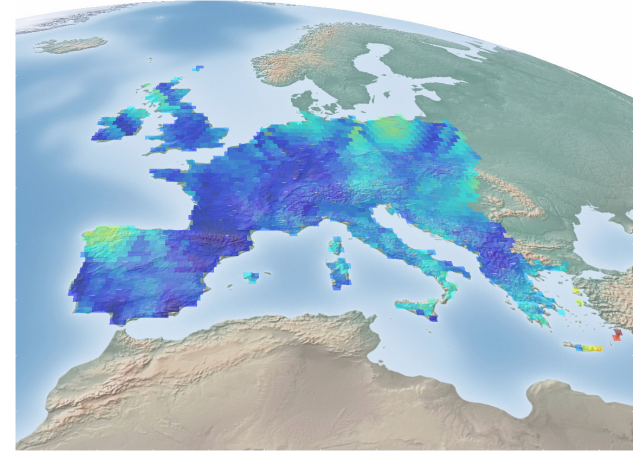

(b)

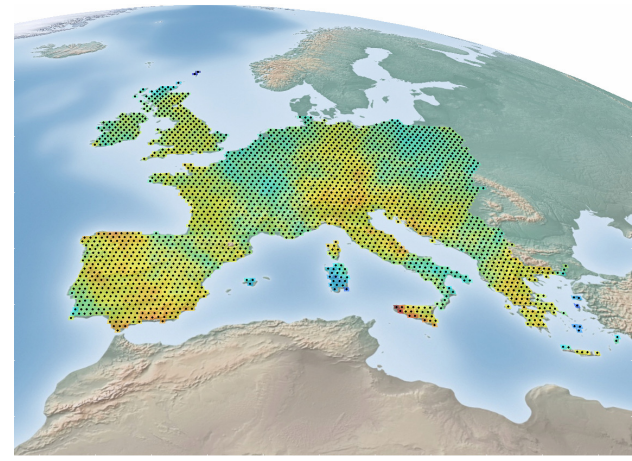

(d)

Figure 14: (a) I3 high heterogeneity (b) I3 low heterogeneity (c) I1 small coverage area (d) I1 wide coverage area

In each category of instances, the number of station $N_{S}$ considered is comprised between 100 and 1000 . The maximum number of beams has been tuned according to the size of the service area : the wider the service area, the higher $n_{\max }$. The number of reflectors has been set to $N_{R}=4$, the number of diameters $N_{W}=2\left(W_{1}=0.35^{\circ}\right.$ et $\left.W_{2}=0.5^{\circ}\right), \kappa=\sqrt{3}$ and $\varepsilon=\frac{1}{2}$. The number of directions has been set to $n_{\text {directions }}=12$. In the results provided below, we present scores obtained by the different algorithms directly in terms of objective function. Note that the experiments were run on a 6 Cores Intel Xeon X5690 @ 3.47 GHz and 24Go of RAM (each run being limited to 2 cores out of the 6 available).

\subsection{Clustering based MILP experiments: comparison of MILP1, MILP2, MILP3}

After optimizing the number of clusters for each instance considered, the three integer linear programs are executed with a timeout of 1500 seconds, which is the maximum acceptable time according to out industrial partner. They are then compared in terms of objective function. The results are depicted in figures 15, 16 and 17. In each figure, the objective function values (aggregate covered traffic) obtained by the MILP1, MILP2 and MILP3 on each instance family $H_{i}$ are plotted for each instance of the family in increasing size order. 


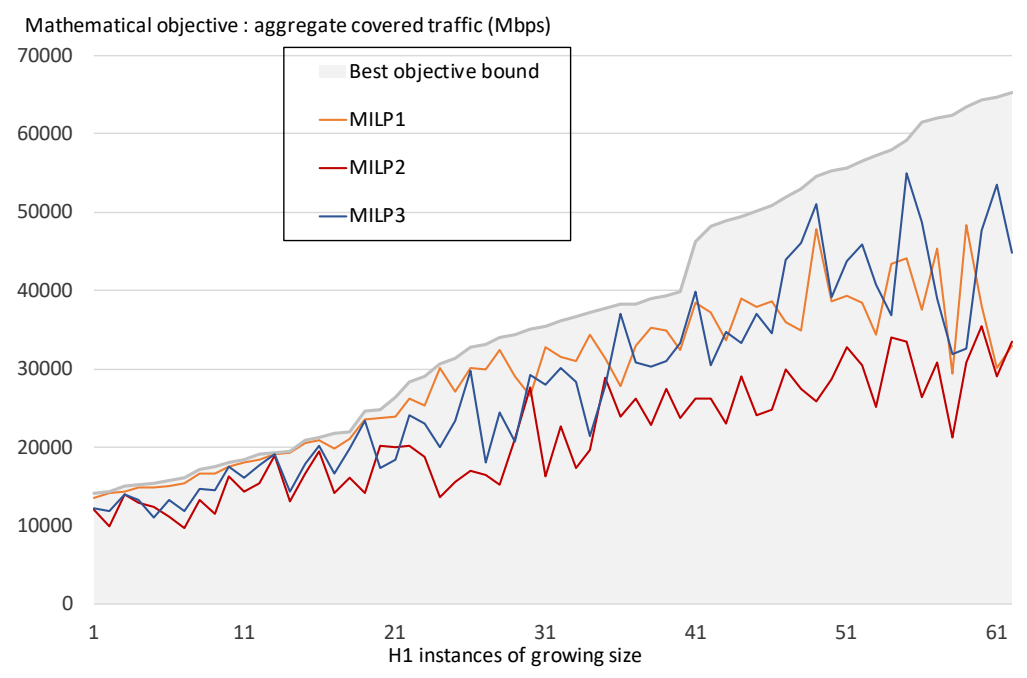

(a)

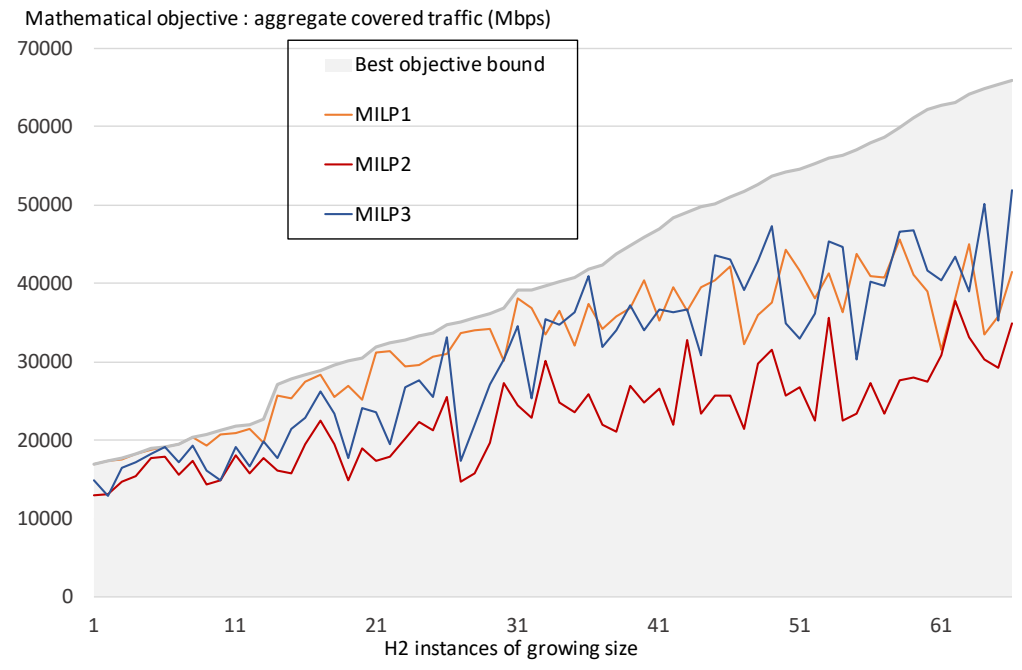

(b)

Figure 15: (a) Aggregate covered traffic for $H_{1}$ (b) Aggregate covered traffic for $H_{2}$ 


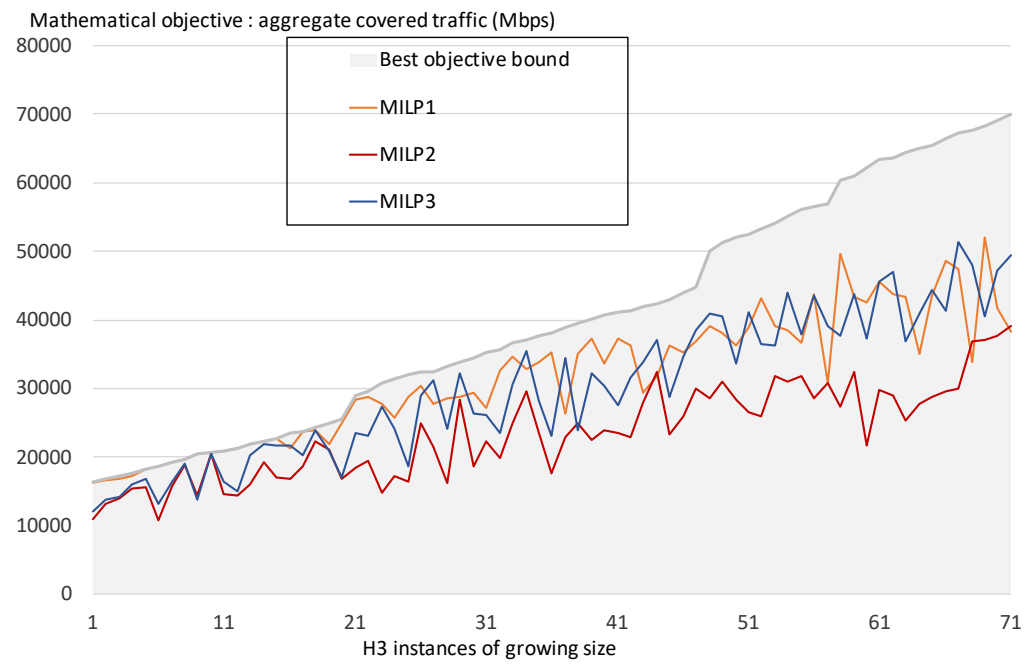

(c)

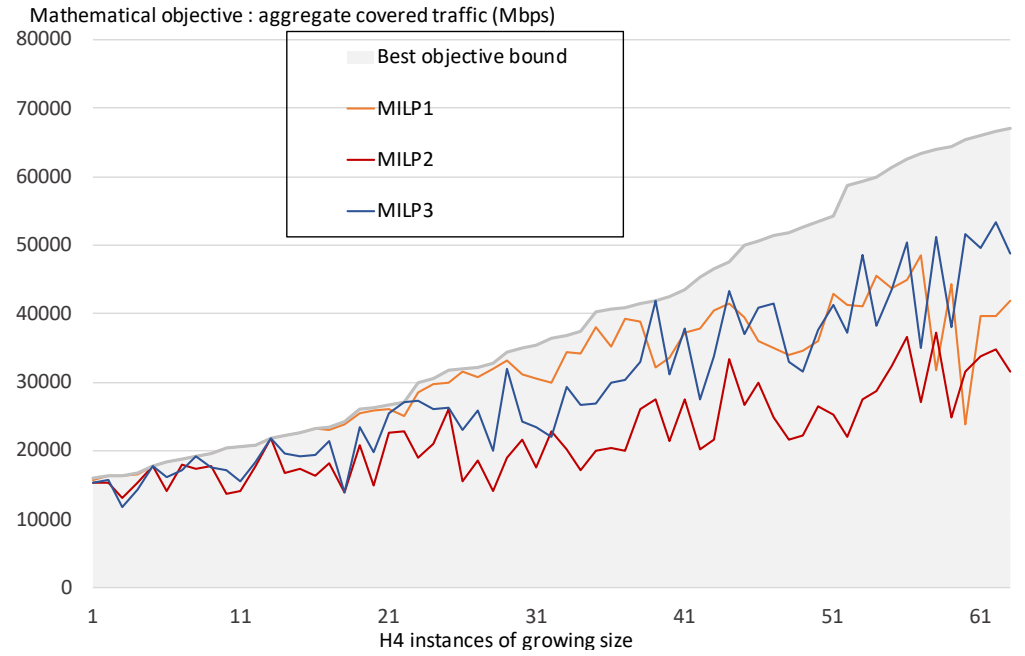

(d)

Figure 16: (c) Aggregate covered traffic for $H_{3}$ (d) Aggregate covered traffic for $H_{4}$ 


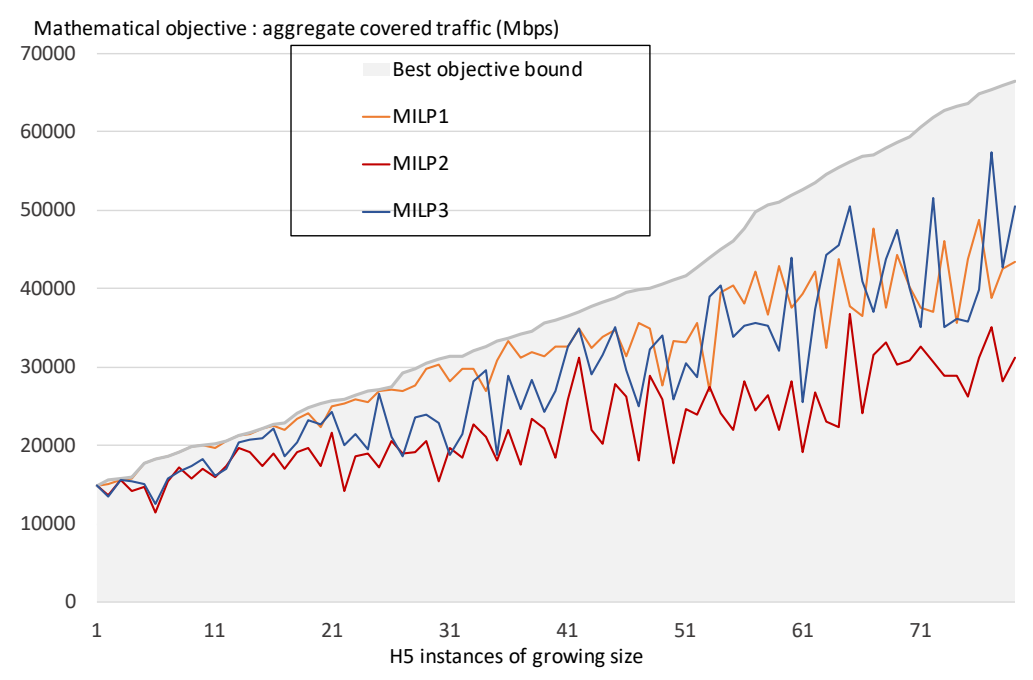

(e)

Figure 17: (e) Aggregate covered traffic for $H_{5}$

As a first preliminary remark, we could not observe a significant influence of the user traffic distribution heterogeneity on the model performances. This is a desirable feature as the proposed MILP-based approach can be used in practice whatever the context whereas the traditional hexagonal regular beam layout is only applicable for uniform traffic demands. A second remark is that the exact clustering MILP2 is outperformed by the two other MILPs : on the complete set of tested instances, we observe a relative difference of $+38.2 \%$ on the solution objectives in favor of the raw linear program MILP1. This observation is directly linked to the number of variables and constraints that is significantly higher in MILP2.

We observe in the figures that MILP1 and MILP3 do not dominate each other in general but that MILP3 tends to outperform MILP1 when the instance size grows. To confirm this observation we generated an additional set of 30 larger instances with a number of stations varying from 300 to 400 and a number of beams ranging from 30 to 40 .

In Table 1, we group all instances regardless of their heterogeneity and we compare the performances of MILP1 and MILP3. We further divide the instances into three categories : small, medium (taken from $H_{1}, \ldots, H_{5}$ ) and large instances (the new set) and we display, for each instance size, the number of considered instances, the average gap $\left(z_{3}-z_{1}\right) / z 1$ where $z 1$ and $z 3$ denote the objective function value reached by MILP1 and MILP3, respectively, and the percentage of instances for which $z 3>z 1$.

\begin{tabular}{|l||c|c|c|}
\hline instance size & number of instances & average gap & percentage of improved instances \\
\hline small & 265 & $-9.95 \%$ & $18.11 \%$ \\
medium & 76 & $8.87 \%$ & $61.86 \%$ \\
large & 40 & $123.67 \%$ & $100 \%$ \\
\hline
\end{tabular}

Table 1: Comparison of MILP1 and MILP3 on small, medium and large instances (1500s)

If we now compare the raw linear programming model MILP1 to the one with heuristic clustering MILP3, we first notice that the latter is less efficient than the former on small instances. Thus, the interest of clustering in is more obvious on wider service zones. This can be explained by the fact that small service areas cannot be split into subregions that are independent in terms of separation constraints for the beams using the same reflector. On the contrary, for independent regions, a significant number of variables can be suppressed in the model thanks to the 
clustering method. Hence for medium regions and, even more, for large regions, the proposed clustering-based method outperforms the raw MILP formulation.

\section{Concluding remarks}

In this article, we considered a beam layout optimization problem for the design of multibeam telecommunication satellites. A previously-proposed mixed integer linear programming formulation based on the linearization process of angular proximity and separation constraints is recalled. A new method for computing an upper bound of the number of required beams to cover a given service area was then described. Finally, we also proposed a generic $k$-means clustering based techniques to break symmetries and reduce the number of variables of the linear program, which lead to two additional MILPs to solve the beam layout optimization problem. The three mixed integer linear programming models were compared on realistic instances. The conclusions of the different experiments conducted are the following: the raw linear program should be used to solve small instances, while the clustering-based heuristic is more adapted to solve medium and large instances, up to thousands end-user stations. Further promising work could consist in hybridizing discrete and continuous beam layout methods by first solving the problem in a pure discrete setting as in [3] where a limited number of possible beam centers is considered and then by adjusting continuously the beam coordinates and reallocating the antenna reflectors by the proposed approach to increase the system's performance.

\section{References}

[1] Alhusseini, M., Azmi, P., Mokari, N., 2019. Optimal joint subcarrier and power allocation for miso-noma satellite networks. Physical Communication 32, 50-61.

[2] Angeletti, P., Lizarraga, J., 2012. Non-regular multibeam coverage payloads for non-uniform traffic demand, in: $2^{\text {nd }}$ ESA Workshop on Advanced Flexible Telecom Payloads.

[3] Camino, J.T., Artigues, C., Houssin, L., Mourgues, S., 2014. A greedy approach combined with graph coloring for non-uniform beam layouts under antenna constraints in multibeam satellite systems, in: Advanced Satellite Multimedia Systems Conference and the 13th Signal Processing for Space Communications Workshop (ASMS/SPSC), pp. 374-381.

[4] Camino, J.T., Artigues, C., Houssin, L., Mourgues, S., 2016. Mixed-integer linear programming for multibeam satellite systems design: Application to the beam layout optimization, in: 2016 Annual IEEE Systems Conference (SysCon), IEEE. pp. 1-6.

[5] Camino, J.T., Artigues, C., Houssin, L., Mourgues, S., 2019. Linearization of euclidean norm dependent inequalities applied to multibeam satellites design. Computational Optimization and Applications 73, 679-705.

[6] Chang, H., 2010. A simple proof of thue's theorem on circle packing. arXiv preprint arXiv:1009.4322 .

[7] Christopoulos, D., Sharma, S., Chatzinotas, S., Krause, J., Ottersten, B., 2015. Coordinated multibeam satellite co-location : The dual satellite paradigm. arXiv preprint arXiv:1503.06981 .

[8] Evans, B., Thompson, P., Kyrgiazos, A., 2012. Irregular beam sizes and non-uniform bandwidth allocation in hts multibeam satellite systems, in: $31^{\text {st }}$ AIAA International Communications Satellite Systems Conference (ICSSC), p. 5617.

[9] Fazlollahi, S., Bungener, S.L., Mandel, P., Becker, G., Marchal, F., 2014. Multi-objectives, multi-period optimization of district energy systems: I. selection of typical operating periods. Computers Chemical Engineering $65,54-66$.

[10] Houssin, L., Artigues, C., Corbel, E., 2011. Frequency allocation problem in a sdma satellite communication system. Computers And Industrial Engineering 61, 346-351. 
[11] Kiatmanaroj, K., Artigues, C., Houssin, L., 2016. On scheduling models for the frequency interval assignment problem with cumulative interferences. Engineering Optimization 48, 740-755.

[12] Kiatmanaroj, K., Artigues, C., Houssin, L., Messine, F., 2013. Frequency assignment in a SDMA satellite communication system with beam decentring feature. Computational Optimization and Applications 56, 439455 .

[13] Liao, K., Guo, D., 2008. A clustering-based approach to the capacitated facility location problem. Transactions in GIS 12, 323-339.

[14] Lin, W., Deng, Z., Fang, Q., Li, N., Han, K., 2017. A new satellite communication bandwidth allocation combined services model and network performance optimization. International Journal of Satellite Communications and Networking 35, 263-277.

[15] Lloyd, S.P., 1982. Least squares quantization in PCM. IEEE Transactions on Information Theory 28, 129-137.

[16] Mahajan, M., Nimbhorkar, P., Varadarajan, K., 2012. The planar k-means problem is np-hard. Theoretical Computer Science 442, 13-21.

[17] Manzour, H., Torabi, A., Pishvaee, M.S., 2013. New heuristic methods for the single-source capacitated multi facility weber problem. The International Journal of Advanced Manufacturing Technology 69, 1569-1579.

[18] Park, U., Kim, H.W., Oh, D.S., Ku, B.J., 2012. A dynamic bandwidth allocation scheme for a multi-spot-beam satellite system. Etri Journal 34, 613-616.

[19] Poulenard, S., Ruellan, M., Roy, B., Riédi, J., Parol, F., Rissons, A., 2014. High altitude clouds impacts on the design of optical feeder link and optical ground station network for future broadband satellite services, in: Free-Space Laser Communication and Atmospheric Propagation XXVI, International Society for Optics and Photonics. Doi: 10.1117/12.2038486.

[20] Santoyo-González, A., Cervelló-Pastor, C., 2018. Latency-aware cost optimization of the service infrastructure placement in 5g networks. Journal of Network and Computer Applications 114, 29-37.

[21] Touya, T., Auroux, D., 2008. Control and topological optimization of a large multibeam array antenna, in: Fifth IASTED International Conference on Antennas, Radar and Wave Propagation, pp. 36-41.

[22] Vidal, O., Verelst, G., Lacan, J., Alberty, E., Radzik, J., Bousquet, M., 2012. Next generation high throughput satellite system, in: 2012 IEEE First AESS European Conference on Satellite Telecommunications (ESTEL), IEEE. pp. 1-7.

[23] Wang, H., Liu, A., Pan, X., Li, J., 2014. Optimization of power allocation for a multibeam satellite communication system with interbeam interference. Journal of Applied Mathematics 2014, doi: 10.1155/2014/469437.

[24] Wang, X., Li, Y., Zhao, S., Zheng, Y., Zhu, Z., Cao, G., 2019. A tradeoff resource allocation based on mf-tdma scheme in the multibeam data relay satellite systems. International Journal of Satellite Communications and Networking 37, 200-212.

[25] You, M., Xiao, Y., Zhang, S., Yang, P., Zhou, S., 2019. Optimal mathematical programming for the warehouse location problem with euclidean distance linearization. Computers \& Industrial Engineering 136, 70-79.

[26] Zhou, C., Mazumder, A., Das, A., Basu, K., Matin-Moghaddam, N., Mehrani, S., Sen, A., 2018. Relay node placement under budget constraint, in: Proceedings of the 19th International Conference on Distributed Computing and Networking, ACM. p. 35.

[27] Zhou, C., Mazumder, A., Das, A., Basu, K., Matin-Moghaddam, N., Mehrani, S., Sen, A., 2019. Relay node placement under budget constraint. Pervasive and Mobile Computing 53, $1-12$. 